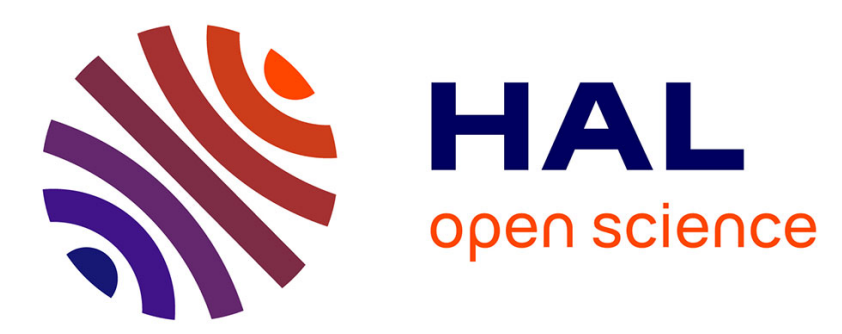

\title{
A density-dependent model of competition for one resource in the chemostat
}

Radhouane Fekih-Salem, Claude Lobry, Tewfik Sari

\section{To cite this version:}

Radhouane Fekih-Salem, Claude Lobry, Tewfik Sari. A density-dependent model of competition for one resource in the chemostat. Mathematical Biosciences, inPress, 286, pp.104-122. 10.1016/j.mbs.2017.02.007 . hal-01359078

\section{HAL Id: hal-01359078 https://hal.science/hal-01359078}

Submitted on 1 Sep 2016

HAL is a multi-disciplinary open access archive for the deposit and dissemination of scientific research documents, whether they are published or not. The documents may come from teaching and research institutions in France or abroad, or from public or private research centers.
L'archive ouverte pluridisciplinaire HAL, est destinée au dépôt et à la diffusion de documents scientifiques de niveau recherche, publiés ou non, émanant des établissements d'enseignement et de recherche français ou étrangers, des laboratoires publics ou privés. 


\title{
A density-dependent model of competition for one resource in the chemostat
}

\author{
Radhouane Fekih-Salem ${ }^{\mathrm{a}, \mathrm{d}, *}$, Claude Lobry ${ }^{\mathrm{b}}$, Tewfik Sari $^{\mathrm{c}, \mathrm{e}}$ \\ ${ }^{a}$ Université de Tunis El Manar, ENIT, LAMSIN, BP 37, Le Belvédère, 1002 Tunis, Tunisie \\ ${ }^{b}$ Université de Nice et MODEMIC, Le Gd Palais Bt6, 2 BD de Cimiez, 06000 Nice, France \\ ${ }^{c}$ IRSTEA, UMR Itap, 361 rue Jean-François Breton, 34196 Montpellier, France \\ ${ }^{d}$ Université de Monastir, ISIMa, BP 49, Av Habib Bourguiba, 5111 Mahdia, Tunisie \\ ${ }^{e}$ Université de Haute Alsace, LMIA, 4 rue des frères Lumière, 68093 Mulhouse, France
}

\begin{abstract}
This paper deals with a two-microbial species model in competition for a single-resource in the chemostat including general intra- and interspecific density-dependent growth rates with distinct removal rates for each species. In order to understand the effects of intra- and interspecific competition, this general model is first studied by determining the conditions of existence and local stability of steady states.

With the same removal rate, the model can be reduced to a planar system and then the global stability results for each steady state are derived. The bifurcations of steady states according to interspecific competition parameters are analyzed in a particular case of density-dependent growth rates which are usually used in the literature.

The operating diagrams show how the model behaves by varying the operating parameters and illustrate the effect of the intra- and interspecific competition on the disappearance of coexistence region and the occurrence of bi-stability region. Concerning the small enough interspecific competition terms, we would shed light on the global convergence towards the coexistence steady state for any positive initial condition. And, as far as the large enough interspecific competition terms, this system exhibits bi-stability with competitive exclusion of one species according to the initial condition.
\end{abstract}

Keywords: Bifurcation, Chemostat, Coexistence, Competition, Density-dependence, Global stability

\section{Introduction}

The chemostat model describes the interactions of microbial species which are competing for a single nutrient, see the monograph of Smith and Waltman [46] for a review. This model is the following one

$$
\left\{\begin{aligned}
\dot{S} & =D\left(S_{i n}-S\right)-\sum_{i=1}^{n} \mu_{i}(S) x_{i} \\
\dot{x}_{i} & =\left[\mu_{i}(S)-D_{i}\right] x_{i}, \quad i=1, \ldots, n
\end{aligned}\right.
$$

where $x_{i}, i=1, \ldots, n$, denotes the concentration of species $i$ and $S$ is the nutrient (substrate) concentration; $S_{\text {in }}$ and $D$ denote, respectively, the concentration of substrate in the feed bottle and the dilution rate of the chemostat; for $i=1, \ldots, n, \mu_{i}(S)$ represents the growth rate of species $i$ and is assumed to be an increasing function. The $D_{i}$ are not necessarily equal to $D$ and can be interpreted as the sum of the dilution rate $D$ and the natural death rate of the species: $D_{i}=D+a_{i}$, where $a_{i} \geqslant 0$. The case $D_{i}<0$, for some $i$, is considered also in the literature. However, this paper focuses only on the case $D_{i} \geqslant D$ for all $i$. It is well known that for (1) only one species can survive at steady state. Actually at a steady state of (1) we must have

$$
\left[\mu_{i}(S)-D_{i}\right] x_{i}=0, \quad i=1, \ldots, n
$$

\footnotetext{
*Corresponding author

Email addresses: radhouene.fekihsalem@isima.rnu.tn (Radhouane Fekih-Salem), lobrinria@wanadoo.fr (Claude Lobry), tewfik.sari@irstea.fr (Tewfik Sari)
} 
If at least two species $x_{i}$ and $x_{j}$, with $i \neq j$ are present at a steady state then $\mu_{i}(S)=D_{i}$ and $\mu_{j}(S)=D_{j}$ are two equations in the single variable $S$ and, in general, cannot have a solution. Hence, in general, besides the washout steady state $\mathcal{E}_{0}$ where $S=S_{\text {in }}$ and $x_{i}=0, i=1, \ldots, n$, where all species are extinct, model (1) can only have steady states $\mathcal{E}_{i}$ where $S=\lambda_{i}$ is a solution of equation $\mu_{i}(S)=D_{i}, x_{i}=\frac{D}{D_{i}}\left(S_{i n}-\lambda_{i}\right)$, and $x_{j}=0$ for $j \neq i$, that is all species except one go to extinction. This steady state is meaningful only if $\lambda_{i}<S_{i n}$.

The $\lambda_{i}$ are called break-even concentrations. If the species are labeled such that $\lambda_{1}<\lambda_{i}$ for all $i>1$, it is well known that the steady state $\mathcal{E}_{1}$ is locally asymptotically stable and all other steady states $\mathcal{E}_{0}$ and $\mathcal{E}_{i}, i>1$, are unstable [46]. Hence, at steady state, only the species with the lowest break-even concentration survives, this is the species which consumes less substrate to attain its steady state. One of the main results for (1) is known as the Competitive Exclusion Principle (CEP), after Hardin [17]. This result states that the locally stable steady state $\mathcal{E}_{1}$ is in fact globally attractive. The CEP was established by several authors under various hypothesis: Hsu et al. [22] showed the global asymptotic stability of $\mathcal{E}_{1}$ in the case of Michaelis-Menten (or Monod) kinetics [36] and the same removal rates $D_{i}$, Hsu [21] extended the study of [22] with different removal rates by using a Lyapunov-LaSalle argument, Wolkowicz and $\mathrm{Lu}$ [50] extended the result of [21] to growth functions which are not necessarily increasing functions of the substrate $S$. The reader may consult $[29,31,43]$ for a more thorough account on the contributions of diverse authors.

Although this theoretical prediction has been corroborated by the experiences of Hansen and Hubell [16], the biodiversity found in nature as well as in waste-water treatment processes and biological reactors seems to contradict the CEP. For example, in aquatic ecosystems, several phytoplankton species competing for some resources can coexist $[26,44]$. The biodiversity is also found in biological reactors with a mixture including at least two competitors for one resource, see [19,45]. This has triggered a lot of mathematical research aimed to extend model (1) to bring theory and observations in better accordance. Different mechanisms of coexistence which were proposed in the literature are the intra- and interspecific competition [1, 11, 28, 51], the flocculation [8, 9, 14, 15] and the density-dependence [32-35]. Several mathematical models $[4,12,13,23-25,30]$ have attempted to understand the effects of an inhibitor on the competition and the coexistence of species in the chemostat. More precisely, competition models of two populations of microorganisms for a single nutrient have been studied with the presence of an inhibitor that affects the most stronger competitor while it is detoxified by the other competitor. See also [5, 27, 39] for other studies of coexistence mechanisms.

In this paper, we will consider models with density-dependent growth functions. The general model is the following extension of (1)

$$
\left\{\begin{array}{l}
\dot{S}=D\left(S_{i n}-S\right)-\sum_{i=1}^{n} \mu_{i}\left(S, x_{1}, \ldots, x_{n}\right) x_{i} \\
\dot{x}_{i}=\left[\mu_{i}\left(S, x_{1}, \ldots, x_{n}\right)-D_{i}\right] x_{i}, \quad i=1, \ldots, n
\end{array}\right.
$$

where the growth function $\mu_{i}\left(S, x_{1}, \ldots, x_{n}\right)$ can depend now not only on the substrate $S$ but also on the species concentrations $x_{i}, i=1, \ldots, n$. The function $\mu_{i}$ is assumed to be increasing in the variable $S$ and decreasing in each variable $x_{j}$. This model was considered in a series of paper by Lobry et al. [18, 32-35]. Now, for (2) it is possible to have a coexistence steady state since at steady state we must have

$$
\left[\mu_{i}\left(S, x_{1}, \ldots, x_{n}\right)-D_{i}\right] x_{i}=0, \quad i=1, \ldots, n .
$$

If all $x_{i}$ are positive then

$$
\mu_{i}\left(S, x_{1}, \ldots, x_{n}\right)=D_{i}, \quad i=1, \ldots, n
$$

is a set of $n$ equations with $n$ variables $x_{i}$ which could have a positive solution $x_{i}=X_{i}(S), i=1, \ldots, n$. Replacing $x_{i}$ by $X_{i}(S)$ in the first equation leads the following equation in the single variable $S$

$$
D\left(S_{i n}-S\right)-\sum_{i=1}^{n} D_{i} X_{i}(S)=0
$$

Solving this equation gives $S$ and then the possibility of a positive steady state.

In [35] the authors considered the case where $\mu_{i}\left(S, x_{i}\right)$ depends only on the concentration of species $i$. They introduced the concept of steady-state characteristic for each species and showed how to use it to give sufficient conditions for coexistence and to determine the asymptotic behavior of the system. Global results were also obtained 
when $D_{i}=D$ for all species [34]. In [8] it is shown that the method of steady-state characteristic is still applicable in the case where both the growth rate $\mu_{i}\left(S, x_{i}\right)$ and the removal rate $D_{i}=d_{i}\left(x_{i}\right)$ of each species depend on the density of the same species. In [1] it is shown that the method of steady-state characteristic permits a quite comprehensive analysis of the model considered in [28], where the growth rate $\mu_{i}(S)$ depends only on $S$ but the removal rate of $x_{i}$ is of the form $D_{i}+a_{i} x_{i}$. The term $a_{i} x_{i}$ corresponds to the so-called crowding effect.

General model (2) was considered in [32] only through numerical simulations. These authors considered a particular situation where the growth functions are of the form

$$
\mu_{i}\left(S, x_{1}, \ldots, x_{n}\right)=v_{i}\left(S, x_{i}+\alpha \sum_{j \neq i} x_{j}\right)
$$

where $\alpha$ is a nonnegative parameter which denotes the interspecific competition. They observed that the coexistence which was predicted in [35], when $\alpha=0$ (only intraspecific competition is present), is still a property of the model when $\alpha$ is small enough but it is no longer the case when $\alpha$ is sufficiently large. The particular case of $(2,3)$ where $n=2$ (two species) and $D_{1}=D_{2}=D$, has been studied theoretically in [7,10]. In these works, the authors gave theoretical explanations for the phenomenon, which was numerically observed in [32], where the coexistence holds for $\alpha$ small enough while the exclusion of one species occurs for $\alpha$ large enough. Growth functions of form (3) and density dependent yields were considered also in [40].

Actually, we have not at our disposal any general mathematical study of (2). Our work can be considered as the first step towards a mathematical study of (2). We consider the two species model

$$
\left\{\begin{array}{l}
\dot{S}=D\left(S_{i n}-S\right)-\mu_{1}\left(S, x_{1}, x_{2}\right) x_{1}-\mu_{2}\left(S, x_{2}, x_{1}\right) x_{2} \\
\dot{x}_{1}=\left[\mu_{1}\left(S, x_{1}, x_{2}\right)-D_{1}\right] x_{1} \\
\dot{x}_{2}=\left[\mu_{2}\left(S, x_{2}, x_{1}\right)-D_{2}\right] x_{2} .
\end{array}\right.
$$

Notice that for the convenience of the notations, the first species variable in $\mu_{i}\left(S, x_{i}, x_{j}\right)$ is the variable $x_{i}$, with the same index $i$. We will give a particular attention to the special case where growth rates are of the form (3) but with different interspecific competition parameters:

$$
\mu_{i}\left(S, x_{i}, x_{j}\right)=v_{i}\left(S, x_{i}+\alpha_{i} x_{j}\right), \text { where } i=1,2, j=1,2, \quad i \neq j
$$

where $\alpha_{1}$ and $\alpha_{2}$ are not necessarily equals. Therefore our study gives an extension of the results in [7, 10] to the case where $D_{1}$ and $D_{2}$ can be different from $D$ and $\alpha_{1}$ and $\alpha_{2}$ can also be different. Our study gives also an extension, in the case $n=2$, of the results in [34,35], where only intraspecific competition occurs, to the case where both intraand interspecific competition occurs, that is, the growth function $\mu_{i}$ depends on both species $x_{1}$ and $x_{2}$, not only on $x_{i}$. In this case $n=2$, we give theoretical explanations for the phenomenon, which was numerically observed in [32], where the coexistence holds when only intraspecific competition occurs whereas it can disappear when the strength of interspecific competition is large enough.

On the other hand, our study describes the operating diagram which shows the stability regions, in dependence of the operating parameters $D$ and $S_{i n}$, when all biological parameters are fixed. This bifurcation diagram is an important tool for the experimentation as discussed in [1, 12, 42, 48, 49].

This paper is organized in the following manner. In Section 2, we present the assumptions on general model (4) and we study the existence conditions of all corresponding steady states. In Section 3, the main result of this paper exhibits the number and local stability of steady states of (4) according to four cases that must be distinguished. In Section 4, we consider the particular case $D_{1}=D_{2}=D$, where model (4) can be reduced to a planar system, and we show global stability results. In Section 5, the particular case (5) is considered in order to show the number and local stability of steady states of (4) according to the relative positions of the interspecific competition parameters of each species. Then, our results are compared with those obtained by Lobry et al. $[34,35]$ in the particular case $\alpha_{1}=\alpha_{2}=0$ and $D_{1}=D_{2}=D$ by using the concept of steady-state characteristics. In Section 6, the operating diagrams depict the existence and the stability of each steady state according to control parameters $D$ and $S_{\text {in }}$. Section 7 is devoted to applying the results found in the case where the density-dependent growth functions are of modified Monod-type (49) or of generalized Monod-type (52). The conclusion is given in Section 8.

For convenience, we use the abbreviations LES for Locally Exponentially Stable steady states and GAS for Globally Asymptotically Stable steady states, in all what follows. 


\section{Assumptions on the model and steady states}

We consider model (4). We make the following assumptions.

(H1) For $i=1,2, j=1,2, i \neq j, \mu_{i}\left(0, x_{i}, x_{j}\right)=0$ and $\mu_{i}\left(S, x_{i}, x_{j}\right)>0$ for all $S>0, x_{1} \geqslant 0$ and $x_{2} \geqslant 0$.

(H2) For $i=1,2, j=1,2, i \neq j, \frac{\partial \mu_{i}}{\partial S}\left(S, x_{i}, x_{j}\right)>0, \frac{\partial \mu_{i}}{\partial x_{i}}\left(S, x_{i}, x_{j}\right) \leqslant 0$ and $\frac{\partial \mu_{i}}{\partial x_{j}}\left(S, x_{i}, x_{j}\right) \leqslant 0$ for all $S>0, x_{1} \geqslant 0$ and $x_{2} \geqslant 0$.

Condition (H1) means that the growth can take place if and only if the substrate is present. Condition (H2) means that the growth rate of each species increases with the concentration of substrate and is inhibited by intra- and interspecific competition. We have the following result:

Proposition 1. For any nonnegative initial condition, the solutions of (4) remain nonnegative and are positively bounded. Moreover, the set

$$
\Omega=\left\{\left(S, x_{1}, x_{2}\right) \in \mathbb{R}_{+}^{3}: S+x_{1}+x_{2} \leqslant S_{\text {in }}\right\}
$$

is positively invariant and is a global attractor for (4).

Proof. Let $S(0) \geqslant 0$, once there exists a first time $t_{0} \geqslant 0$ such that $S\left(t_{0}\right)=0$, we have

$$
\dot{S}\left(t_{0}\right)=D S_{\text {in }}>0 .
$$

Hence $S(t) \geqslant 0$ for all $t \geqslant t_{0}$. Since $S(t) \geqslant 0$ for all $t \in\left[0, t_{0}\right]$, thus $S(t) \geqslant 0$ for all $t \geqslant 0$. In the same manner,

$$
x_{i}=0 \Rightarrow \dot{x}_{i}=0 \quad i=1,2 \text {, }
$$

and hence $x_{i}(t) \geqslant 0$ for all $t \geqslant 0$. Therefore, the solutions of (4) remain nonnegative. Denote $z=S+x_{1}+x_{2}$. So, we have

$$
\dot{z}=D\left(S_{\text {in }}-z\right)-a_{1} x_{1}-a_{2} x_{2},
$$

where $a_{1}=D_{1}-D \geqslant 0$ and $a_{2}=D_{2}-D \geqslant 0$. Therefore, $\dot{z} \leqslant D\left(S_{\text {in }}-z\right)$. Then,

$$
z(t) \leqslant S_{\text {in }}+\left(z(0)-S_{\text {in }}\right) e^{-D t}, \text { for all } t \geqslant 0 .
$$

Hence

$$
z(t) \leqslant \max \left(z(0), S_{\text {in }}\right) \text { for all } t \geqslant 0 .
$$

Therefore, the solutions of (4) are positively bounded and are defined for all $t \geqslant 0$. From (6), it can be deduced that the set $\Omega$ is positively invariant and is a global attractor for (4).

The steady states of (4) are the solutions of the set of equations

$$
\left\{\begin{array}{l}
0=D\left(S_{\text {in }}-S\right)-\mu_{1}\left(S, x_{1}, x_{2}\right) x_{1}-\mu_{2}\left(S, x_{2}, x_{1}\right) x_{2} \\
0=\left[\mu_{1}\left(S, x_{1}, x_{2}\right)-D_{1}\right] x_{1} \\
0=\left[\mu_{2}\left(S, x_{2}, x_{1}\right)-D_{2}\right] x_{2} .
\end{array}\right.
$$

Therefore, (4) has the following types of steady states:

- $\mathcal{E}_{0}=\left(S_{\text {in }}, 0,0\right)$, called the washout, where both populations are extinct: $x_{1}=x_{2}=0$. This steady state always exists.

- $\mathcal{E}_{1}=\left(\tilde{S}_{1}, \tilde{x}_{1}, 0\right)$, where second population is extinct: $x_{2}=0$ and $\tilde{x}_{1}>0$.

- $\mathcal{E}_{2}=\left(\tilde{S}_{2}, 0, \tilde{x}_{2}\right)$, where first population is extinct: $x_{1}=0$ and $\tilde{x}_{2}>0$.

- $\mathcal{E}^{*}=\left(S^{*}, x_{1}^{*}, x_{2}^{*}\right)$, where both populations survive: $x_{1}^{*}>0, x_{2}^{*}>0$. 
The components $S=\tilde{S}_{i}$ and $x=\tilde{x}_{i}$ of a boundary steady state $\mathcal{E}_{i}$ are the solutions of (7) with $x_{i}>0$ and $x_{j}=0, j \neq i$. Therefore $\tilde{S}_{i}$ and $\tilde{x}_{i}$ are the solutions of equations

$$
\begin{aligned}
& D\left(S_{i n}-\tilde{S}_{i}\right)=D_{i} \tilde{x}_{i} \\
& \mu_{i}\left(\tilde{S}_{i}, \tilde{x}_{i}, 0\right)=D_{i} .
\end{aligned}
$$

From (8) we have

$$
\tilde{S}_{i}=S_{\text {in }}-\frac{D_{i}}{D} \tilde{x}_{i}
$$

Replacing $\tilde{S}_{i}$ by this expression in (9) we see that $x_{i}=\tilde{x}_{i}$ must be a solution of

$$
\mu_{i}\left(S_{\text {in }}-\frac{D_{i}}{D} x_{i}, x_{i}, 0\right)=D_{i}
$$

$\tilde{S}_{i}$ is positive if and only if $\tilde{x}_{i}<D S_{i n} / D_{i}$, that is to say, (11) has a solution in the interval $\left(0, D S_{\text {in }} / D_{i}\right)$. Let $\psi_{i}$ be the function defined by

$$
\psi_{i}\left(x_{i}\right):=\mu_{i}\left(S_{\text {in }}-\frac{D_{i}}{D} x_{i}, x_{i}, 0\right)-D_{i}, \quad i=1,2 .
$$

From (H1) and (H2) we know that the function $\psi_{i}$ is decreasing from $\psi_{i}(0)=\mu_{i}\left(S_{i n}, 0,0\right)-D_{i}$ to $\psi_{i}\left(D S_{i n} / D_{i}\right)=-D_{i}$. Thus, there exists an $x_{i}=\tilde{x}_{i} \in\left(0, D S_{i n} / D_{i}\right)$ satisfying (11) if and only if

$$
\mu_{i}\left(S_{i n}, 0,0\right)>D_{i}
$$

holds. If such an $\tilde{x}_{i}$ exists then it is unique. Therefore, we obtain the following result which gives the condition of existence of a boundary steady state $\mathcal{E}_{i}$.

Proposition 2. Let $\mathcal{E}_{i}$ be a steady state of (4) with $\tilde{x}_{i}>0$ and $x_{j}=0, j \neq i$. Then $\tilde{S}_{i}$ is given by (10) where $x_{i}=\tilde{x}_{i}$ is the solution of (11). This steady state exists if and only if (13) holds. If it exists then it is unique.

The components $S=S^{*}, x_{1}=x_{1}^{*}$ and $x_{2}=x_{2}^{*}$ of a coexistence steady state $\mathcal{E}^{*}$ must be the solutions of (7) with $x_{1}>0$ and $x_{2}>0$. Therefore $S^{*}, x_{1}^{*}$ and $x_{2}^{*}$ are the solutions of the set of equations

$$
\begin{aligned}
D\left(S_{\text {in }}-S\right) & =D_{1} x_{1}+D_{2} x_{2} \\
\mu_{1}\left(S, x_{1}, x_{2}\right) & =D_{1} \\
\mu_{2}\left(S, x_{2}, x_{1}\right) & =D_{2} .
\end{aligned}
$$

Hence, from (14) we see that $S^{*}$ is given by

$$
S^{*}=S_{i n}-\frac{D_{1}}{D} x_{1}^{*}-\frac{D_{2}}{D} x_{2}^{*}
$$

Replacing $S^{*}$ by this expression in $(15,16)$ we see that $\left(x_{1}=x_{1}^{*}, x_{2}=x_{2}^{*}\right)$ must be a solution of

$$
\left\{\begin{array}{l}
f_{1}\left(x_{1}, x_{2}\right)=0 \\
f_{2}\left(x_{1}, x_{2}\right)=0
\end{array}\right.
$$

where

$$
f_{i}\left(x_{1}, x_{2}\right):=\mu_{i}\left(S_{i n}-\frac{D_{1}}{D} x_{1}-\frac{D_{2}}{D} x_{2}, x_{i}, x_{j}\right)-D_{i}, \quad \text { for } \quad i=1,2, j=1,2, \quad i \neq j .
$$

The functions (19) are defined on the set

$$
M=\left\{\left(x_{1}, x_{2}\right) \in \mathbb{R}_{+}^{2}: \frac{D_{1}}{D} x_{1}+\frac{D_{2}}{D} x_{2} \leqslant S_{\text {in }}\right\} .
$$

$S^{*}$ is positive if and only if $\frac{D_{1}}{D} x_{1}^{*}+\frac{D_{2}}{D} x_{2}^{*}<S_{\text {in }}$, that is to say, (18) has a solution in the interior $M^{o}$ of $M$, defined by (20). To solve (18) in this open $M^{o}$, we need the following result: 
Lemma 1. Assume that (13) holds for $i=1,2$ and let $\tilde{x}_{i}$ be a solution of (11). The equation $f_{i}\left(x_{1}, x_{2}\right)=0$ defines a smooth decreasing function

$$
F_{i}:\left[0, \tilde{x}_{i}\right] \rightarrow \mathbb{R}, \quad x_{i} \mapsto F_{i}\left(x_{i}\right)
$$

such that $F_{i}\left(\tilde{x}_{i}\right)=0$ and the graph $\gamma_{i}$ of $F_{i}$ lies in $M^{o}$ (see Figure 1). More precisely, $\left(x_{1}, F_{1}\left(x_{1}\right)\right) \in M^{o}[$ resp. $\left.\left(F_{2}\left(x_{2}\right), x_{2}\right) \in M^{o}\right]$ for all $x_{i} \in\left(0, \tilde{x}_{i}\right)$.
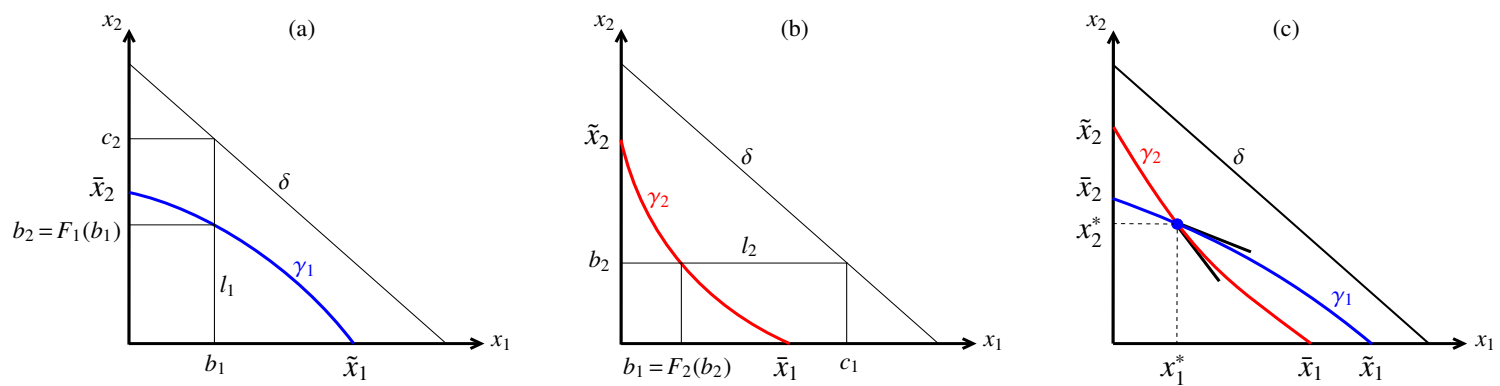

Figure 1: (a) Definition of the function $F_{1}$. (b) Definition of the function $F_{2}$. (c): A blue positive steady state. In all figures, the curves $\gamma_{1}$ and $\gamma_{2}$ are in blue and red, respectively.

Proof. Let us prove that each line $l_{i}$ defined by $x_{i}=b_{i}$ meets the set $f_{i}=0$ exactly once if $0 \leqslant b_{i} \leqslant \tilde{x}_{i}$ (and the intersection belongs to $M^{o}$ ) and not at all if $b_{i}>\tilde{x}_{i}$, (see Figure 1). Indeed, let $x_{i}=b_{i}$ be fixed and for $i=1,2$, $j=1,2, i \neq j$. The line $l_{i}$ intersects the line $\delta$ defined by

$$
\frac{D_{i}}{D} x_{i}+\frac{D_{j}}{D} x_{j}=S_{i n}
$$

at point $x_{j}=c_{j}$ where

$$
c_{j}=\frac{D}{D_{j}} S_{i n}-\frac{D_{i}}{D_{j}} b_{i}
$$

From (H1) and (H2) we see that the function $x_{j} \mapsto \mu_{i}\left(S_{i n}-\frac{D_{i}}{D} b_{i}-\frac{D_{j}}{D} x_{j}, b_{i}, x_{j}\right)$ is decreasing from $\mu_{i}\left(S_{i n}-\frac{D_{i}}{D} b_{i}, b_{i}, 0\right)$ for $x_{j}=0$ to 0 for $x_{j}=c_{j}$. Therefore, there exists a unique $b_{j} \in\left[0, c_{j}\right)$ such that $\mu_{i}\left(S_{i n}-\frac{D_{i}}{D} b_{i}-\frac{D_{j}}{D} b_{j}, b_{i}, b_{j}\right)=D_{i}$, that is $f_{i}\left(b_{1}, b_{2}\right)=0$, if and only if

$$
\mu_{i}\left(S_{\text {in }}-\frac{D_{i}}{D} b_{i}, b_{i}, 0\right)-D_{i} \geqslant 0
$$

which is equivalent to $b_{i} \leqslant \tilde{x}_{i}$ since the function $\psi_{i}$ defined by (12) is decreasing and vanishes at $\tilde{x}_{i}$. Therefore, we have shown that for all $b_{i} \in\left[0, \tilde{x}_{i}\right]$ there exists a unique $b_{j} \in\left[0, c_{j}\right)$ such that $f_{i}\left(b_{1}, b_{2}\right)=0$. We define the function $F_{i}$ by $b_{j}=F_{i}\left(b_{i}\right)$. The graph $\gamma_{i}$ of this function lies in $M^{o}$. By the implicit function theorem, the function $F_{i}$ is smooth and decreasing. Indeed, using (H2), we deduce that the partial derivatives of $f_{i}$ are

$$
\frac{\partial f_{i}}{\partial x_{1}}=-\frac{D_{1}}{D} \frac{\partial \mu_{i}}{\partial S}+\frac{\partial \mu_{i}}{\partial x_{1}}<0, \quad \frac{\partial f_{i}}{\partial x_{2}}=-\frac{D_{2}}{D} \frac{\partial \mu_{i}}{\partial S}+\frac{\partial \mu_{i}}{\partial x_{2}}<0 .
$$

Therefore, the function $F_{i}$ is smooth and its derivative is given by

$$
F_{i}^{\prime}=-\frac{\frac{D_{i}}{D} \frac{\partial \mu_{i}}{\partial S}-\frac{\partial \mu_{i}}{\partial x_{i}}}{\frac{D_{j}}{D} \frac{\partial \mu_{i}}{\partial S}-\frac{\partial \mu_{i}}{\partial x_{j}}}
$$

which is negative. Thus $F_{i}$ is decreasing.

Remark 1. Notice that $F_{i}:\left[0, \tilde{x}_{i}\right] \rightarrow\left[0, \bar{x}_{j}\right], x_{i} \mapsto F_{i}\left(x_{i}\right)$, where $\bar{x}_{j}=F_{i}(0)$ is the unique solution of

$$
\mu_{i}\left(S_{i n}-\frac{D_{j}}{D} x_{j}, 0, x_{j}\right)=D_{i}
$$


Using the definitions (19) of $f_{1}$ and $f_{2}$ one sees that $\tilde{x}_{1}, \tilde{x}_{2}$ which are the solutions of (11) and $\bar{x}_{1}, \bar{x}_{2}$ which are the solutions of (22) are simply the solutions of the following equations

$$
f_{1}\left(\tilde{x}_{1}, 0\right)=0, \quad f_{2}\left(0, \tilde{x}_{2}\right)=0, \quad f_{1}\left(0, \bar{x}_{2}\right)=0, \quad f_{2}\left(\bar{x}_{1}, 0\right)=0 .
$$

These quantities represent the coordinates of the intersections of the curves $\gamma_{1}$ and $\gamma_{2}$ with the coordinates axes. Their relative positions play a major role in the behavior of the system.

The following four cases must be distinguished (see Figures 2 and 3):

$$
\begin{array}{lll}
\text { Case 1: } \bar{x}_{1}>\tilde{x}_{1} \quad \text { and } \quad \bar{x}_{2}>\tilde{x}_{2}, \quad \text { Case 2: } \bar{x}_{1}<\tilde{x}_{1} \quad \text { and } \quad \bar{x}_{2}<\tilde{x}_{2}, \\
\text { Case 3: } \bar{x}_{1}<\tilde{x}_{1} \quad \text { and } \quad \bar{x}_{2}>\tilde{x}_{2}, \quad \text { Case 4: } \bar{x}_{1}>\tilde{x}_{1} \quad \text { and } \quad \bar{x}_{2}<\tilde{x}_{2} .
\end{array}
$$
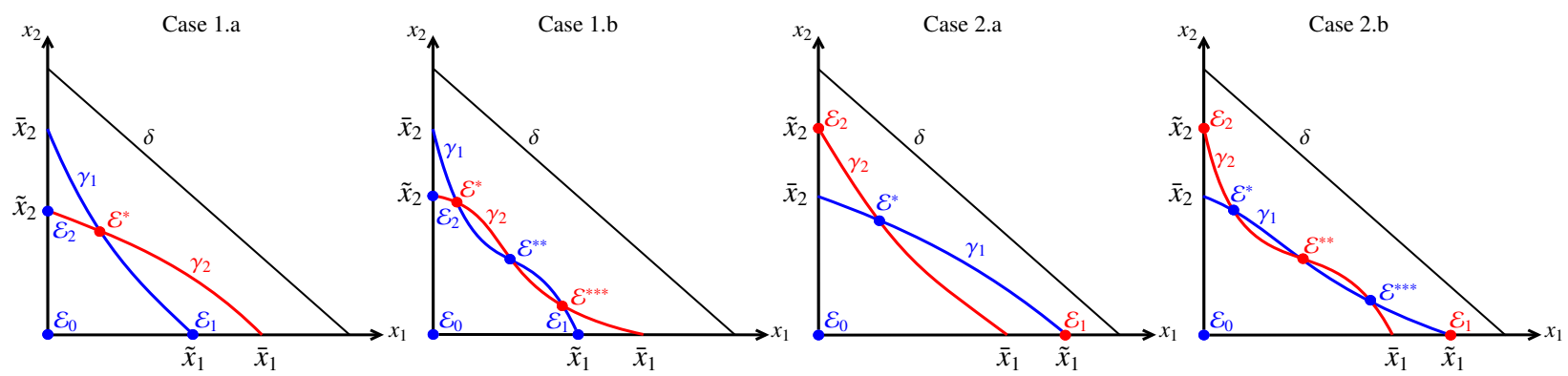

Figure 2: Case 1: $\bar{x}_{1}>\tilde{x}_{1}$ and $\bar{x}_{2}>\tilde{x}_{2}$ : (a) unique intersection, (b) an odd number of intersections. Case $2: \bar{x}_{1}<\tilde{x}_{1}$ and $\bar{x}_{2}<\tilde{x}_{2}$ : (a) unique intersection, (b) an odd number of intersections.
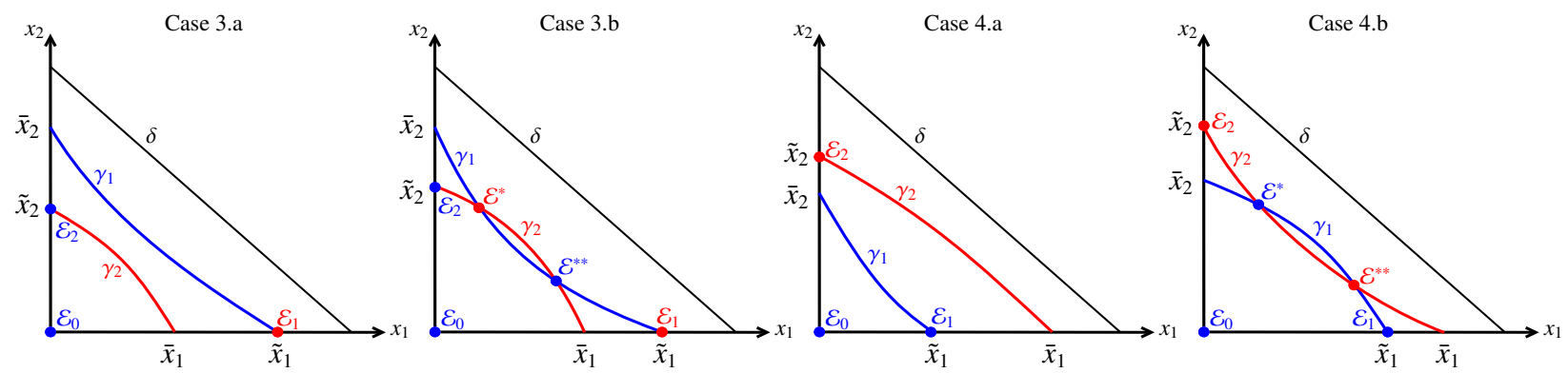

Figure 3: Case $3: \bar{x}_{1}<\tilde{x}_{1}$ and $\bar{x}_{2}>\tilde{x}_{2}$ : (a) no intersection, (b) an even number of intersections. Case $4: \bar{x}_{1}>\tilde{x}_{1}$ and $\bar{x}_{2}<\tilde{x}_{2}$ : (a) no intersection, (b) an even number of intersections.

As a consequence of the previous lemma, we obtain the following result which gives the condition of existence of a positive steady state $\mathcal{E}^{*}$.

Proposition 3. A positive steady state $\mathcal{E}^{*}=\left(S^{*}, x_{1}^{*}, x_{2}^{*}\right)$ of (4) exists if and only if the curves $\gamma_{1}$ and $\gamma_{2}$ have a positive intersection, that is, $\left(x_{1}^{*}, x_{2}^{*}\right)$ is a positive solution of equations

$$
x_{2}=F_{1}\left(x_{1}\right) \text { and } x_{1}=F_{2}\left(x_{2}\right) \text {. }
$$

Then $S^{*}$ is given by (17). If Case 1 or Case 2 hold then a positive steady state exists. This condition of existence of the positive steady state is not necessary, and if it exists the positive steady state is not unique.

Proof. By (18) and Lemma 1, a positive steady state $\mathcal{E}^{*}=\left(S^{*}, x_{1}^{*}, x_{2}^{*}\right)$ exists if and only if the curves $\gamma_{1}$ and $\gamma_{2}$ have a positive intersection $\left(x_{1}^{*}, x_{2}^{*}\right)$. If the intersection exists then it lies in $M^{o}$, so that

$$
\frac{D_{1}}{D} x_{1}^{*}+\frac{D_{2}}{D} x_{2}^{*}<S_{i n}
$$


Hence, $S^{*}$, which is defined by (17) is positive. The sufficient conditions of intersection given by Case 1 or Case 2 are illustrated in Figure 2. The fact that these sufficient conditions of existence of the positive steady state are not necessary is illustrated by Figure 3, Cases 3.b and 4.b. The fact that the positive steady state is not unique, if it exists, is illustrated in Figure 2, Cases 1.b and 2.b, and Figure 3, Cases 3.b and 4.b .

We restrict our attention to the generic situation, where all intersections of curves $\gamma_{1}$ and $\gamma_{2}$ are transverse. We give the following definitions:

Definition 1. A positive steady state $\mathcal{E}^{*}=\left(S^{*}, x_{1}^{*}, x_{2}^{*}\right)$ of $(4)$ is said to be blue [resp. red] if and only if, on the right of $\left(x_{1}^{*}, x_{2}^{*}\right)$, the tangent of $\gamma_{1}$ at point $\left(x_{1}^{*}, x_{2}^{*}\right)$ is above [resp. under] the tangent of $\gamma_{2}$ at point $\left(x_{1}^{*}, x_{2}^{*}\right)$, see Figure 1(c).

The positive steady states are alternatively red and blue and are represented in red and blue colors respectively in Figures 2 and 3. We have the following characterization of red and blue positive steady states.

Lemma 2. A positive steady state $\mathcal{E}^{*}=\left(S^{*}, x_{1}^{*}, x_{2}^{*}\right)$ is blue if and only if $F_{1}^{\prime}\left(x_{1}^{*}\right) F_{2}^{\prime}\left(x_{2}^{*}\right)<1$. It is red if and only if $F_{1}^{\prime}\left(x_{1}^{*}\right) F_{2}^{\prime}\left(x_{2}^{*}\right)>1$.

Proof. The curves $\gamma_{1}$ and $\gamma_{2}$ are the graphs of the functions $x_{1} \mapsto F_{1}\left(x_{1}\right)$ and $x_{1} \mapsto F_{2}^{-1}\left(x_{1}\right)$, respectively, where $F_{2}^{-1}$ is the inverse function of $F_{2}$. Therefore, the positive steady state is blue if and only if $F_{1}^{\prime}\left(x_{1}^{*}\right)>\left(F_{2}^{-1}\right)^{\prime}\left(x_{1}^{*}\right)=\frac{1}{F_{2}^{\prime}\left(x_{2}^{*}\right)}$. Since $F_{2}^{\prime}\left(x_{2}^{*}\right)<0$ this condition is equivalent to $F_{1}^{\prime}\left(x_{1}^{*}\right) F_{2}^{\prime}\left(x_{2}^{*}\right)<1$. The same proof holds for red positive steady states.

\section{Main result}

Our aim is to show the following result:

Theorem 1. Assume that (H1), (H2) and (13), for $i=1,2$ hold.

1. Blue positive steady states are unstable. If for all $S, x_{1}, x_{2}$,

$$
\frac{\partial \mu_{1}}{\partial x_{1}}<\frac{\partial \mu_{1}}{\partial x_{2}} \quad \text { and } \quad \frac{\partial \mu_{2}}{\partial x_{2}}<\frac{\partial \mu_{2}}{\partial x_{1}}
$$

hold, or $D_{1}=D_{2}=D$, then red positive steady states are LES, that is to say, positive steady states are alternatively unstable and LES.

2. If Case 1 holds, the system can have generically an odd number of positive steady states, while $\mathcal{E}_{1}$ and $\mathcal{E}_{2}$ are unstable. The positive steady states at the left-hand end and right-hand end are red (see Figure 2, Case 1). If, in addition, for all $S, x_{1}, x_{2}$,

$$
\frac{\partial \mu_{1}}{\partial x_{1}}<\frac{D_{1}}{D_{2}} \frac{\partial \mu_{1}}{\partial x_{2}} \quad \text { and } \quad \frac{\partial \mu_{2}}{\partial x_{2}}<\frac{D_{2}}{D_{1}} \frac{\partial \mu_{2}}{\partial x_{1}}
$$

hold, then the positive steady state is unique (see Figure 2, Case 1.a).

3. If Case 2 holds, the system can have generically an odd number of positive steady states, while $\mathcal{E}_{1}$ and $\mathcal{E}_{2}$ are LES. The positive steady states at the left-hand end and right-hand end are blue (see Figure 2, Case 2). If, in addition, for all $S, x_{1}, x_{2}$,

$$
\frac{\partial \mu_{1}}{\partial x_{1}}>\frac{D_{1}}{D_{2}} \frac{\partial \mu_{1}}{\partial x_{2}} \quad \text { and } \quad \frac{\partial \mu_{2}}{\partial x_{2}}>\frac{D_{2}}{D_{1}} \frac{\partial \mu_{2}}{\partial x_{1}}
$$

hold, then $\mathcal{E}^{*}$ is unique and unstable (see Figure 2, Case 2.a).

4. If Case 3 [resp. Case 4] holds, then generically the system has no positive steady state or an even number of positive steady states where $\mathcal{E}_{1}$ is LES [resp. unstable] and $\mathcal{E}_{2}$ is unstable [resp. LES]. The positive steady state at the right-hand [resp. left-hand] end, if it exists, is blue (see Figure 3). If, in addition, (27) or (28) hold, then the system has no positive steady state (see Figure 3, Case 3.a [resp. Case 4.a]).

The proof which uses some results given hereafter is postponed until the end of this section. 
Remark 2. When $D_{1}=D_{2}=D$, the stability of positive steady states is completely characterized: a positive steady state is LES if and only if it is red. When $D_{i}$ are distinct, we do not have at our disposal a necessary and sufficient condition for stability: the complete characterization of local behavior of $\mathcal{E}^{*}$ remains an open problem. This problem is solved when conditions (26) hold since in this case we know also that a positive steady state is LES if and only if it is red. However, we were not able to find an example where $D_{i}$ are distinct and (26) does not hold, for which there exists a red positive steady state which is unstable. This question deserves further investigations.

We emphasize on the two following particular situations: Theorem 1 asserts that if Case 2 holds and conditions (28) are satisfied then $\mathcal{E}^{*}$ exists, is unique and is unstable. It asserts also that if Case 1 holds and both conditions (26) and (27) are satisfied then $\mathcal{E}^{*}$ exists, is unique and is LES. These properties deserve the biological interpretations given in the following remark.

Remark 3. Since the partial derivatives $\frac{\partial \mu_{i}}{\partial x_{j}}, i, j=1,2$ are nonpositive, conditions (28) are equivalent to

$$
0 \leqslant-\frac{\partial \mu_{1}}{\partial x_{1}}<\frac{D_{1}}{D_{2}}\left(-\frac{\partial \mu_{1}}{\partial x_{2}}\right) \quad \text { and } \quad 0 \leqslant-\frac{\partial \mu_{2}}{\partial x_{2}}<\frac{D_{2}}{D_{1}}\left(-\frac{\partial \mu_{2}}{\partial x_{1}}\right)
$$

which means that the intraspecific competition in each population of micro-organisms, measured by the partial derivative $\left(-\frac{\partial \mu_{i}}{\partial x_{i}}\right)$, is dominated by the interspecific competition, measured by the partial derivative $\left(-\frac{\partial \mu_{i}}{\partial x_{j}}\right), i=1,2, j=1,2$, $i \neq j$. If these conditions are satisfied then Theorem 1 asserts that, if the positive steady state exists, it is unique and unstable.

Note also that conditions (26) together with conditions (27) are equivalent to

$$
-\frac{\partial \mu_{1}}{\partial x_{1}}>\max \left(\frac{D_{1}}{D_{2}}, 1\right)\left(-\frac{\partial \mu_{1}}{\partial x_{2}}\right) \geqslant 0 \quad \text { and } \quad-\frac{\partial \mu_{2}}{\partial x_{2}}>\max \left(\frac{D_{2}}{D_{1}}, 1\right)\left(-\frac{\partial \mu_{2}}{\partial x_{1}}\right) \geqslant 0 .
$$

Hence, Theorem 1 asserts that in the case where the intraspecific competition is dominant with respect to interspecific competition then, if the positive steady state exists, it is unique and LES. Note that conditions (26) and (27) cannot hold if $\frac{\partial \mu_{1}}{\partial x_{1}}=0$ or $\frac{\partial \mu_{2}}{\partial x_{2}}=0$. On the other hand, conditions (28) cannot hold if $\frac{\partial \mu_{1}}{\partial x_{2}}=0$ or $\frac{\partial \mu_{2}}{\partial x_{1}}=0$.

In order to show Theorem 1, we need the following three propositions whose proofs are postponed to Appendix A. Under conditions (27) or (28), the first proposition shows that the positive steady state is unique, if it exists.

Proposition 4. If (27) holds then, if Case 1 holds, the positive steady state exists and is unique and, if Case 3 or Case 4 hold, there is no positive steady state. This condition is incompatible with Case 2.

If (28) holds then, if Case 2 holds, the positive steady state exists and is unique and, if Case 3 or Case 4 hold, there is no positive steady state. This condition is incompatible with Case 1.

The second proposition determines the local asymptotic behavior of steady states $\mathcal{E}_{0}$ and $\mathcal{E}_{i}, i=1,2$ of (4).

Proposition 5. $\mathcal{E}_{0}$ is LES if and only if $\mu_{i}\left(S_{i n}, 0,0\right)<D_{i}, i=1,2$, that is, $\mathcal{E}_{1}$ and $\mathcal{E}_{2}$ do not exist. For $i=1,2, \mathcal{E}_{i}$ is LES if and only if $\tilde{x}_{i}>\bar{x}_{i}$.

The third proposition gives sufficient conditions for stability, and instability of steady states $\mathcal{E}^{*}$ of (4) when the $D_{i}$ are distinct. When they are equal to $D$, we have a necessary and sufficient condition for stability at our disposal.

Proposition 6. Let $\mathcal{E}^{*}=\left(S^{*}, x_{1}^{*}, x_{2}^{*}\right)$ be a positive steady state.

1. If $F_{1}^{\prime}\left(x_{1}^{*}\right) F_{2}^{\prime}\left(x_{2}^{*}\right)<1$, then $\mathcal{E}^{*}$ is unstable.

2. If (26) holds or $D_{1}=D_{2}=D$, then $\mathcal{E}^{*}$ is LES if and only if $F_{1}^{\prime}\left(x_{1}^{*}\right) F_{2}^{\prime}\left(x_{2}^{*}\right)>1$.

Proof of Theorem 1. From item 1 of Proposition 6 and Lemma 2, it follows that blue positive steady states are unstable. From item 2 of Proposition 6 and Lemma 2, if (26) holds or $D_{1}=D_{2}=D$, then it follows that red positive steady states are LES. This proves item 1 of the theorem.

If Case 1 [resp. Case 2] holds, then according to Proposition 3, there exists at least one positive steady state. Using Proposition 5, we deduce that $\mathcal{E}_{1}$ and $\mathcal{E}_{2}$ exist and are unstable [resp. LES]. The steady states at the left-hand end 
and right-hand end are red [resp. blue], since on the right of the corresponding intersections of curves $\gamma_{1}$ and $\gamma_{2}$, the tangent of $\gamma_{1}$ is under [resp. above] the tangent of $\gamma_{2}$. If (27) [resp. (28)] holds, then using Proposition 4, we deduce that $\mathcal{E}^{*}$ is the unique positive steady state [resp. and is unstable]. If (27) [resp. (28)] does not hold, then in the generic case, the curves $\gamma_{1}$ and $\gamma_{2}$ can have an odd number of intersections. This proves items 2 and 3 of the theorem.

If Case 3 [resp. Case 4] holds, then generically the curves $\gamma_{1}$ and $\gamma_{2}$ have no intersection or an even number of intersections. From Proposition 5, $\mathcal{E}_{1}$ is LES [resp. unstable] and $\mathcal{E}_{2}$ is unstable [resp. LES]. If (27) or (28) hold, then using Proposition 4, we deduce that the system has no positive steady state. The positive steady state at the right-hand [resp. left-hand] end is blue, since on the right of the corresponding intersection of curves $\gamma_{1}$ and $\gamma_{2}$, the tangent of $\gamma_{1}$ is above the tangent of $\gamma_{2}$. This proves item 4 of the theorem.

\section{Same dilution rates}

In this section, we consider the particular case of (4) where $D_{1}=D_{2}=D$. In this case, conditions (26) and (27) are identical and conditions (28) can be written

$$
\frac{\partial \mu_{1}}{\partial x_{1}}>\frac{\partial \mu_{1}}{\partial x_{2}} \quad \text { and } \quad \frac{\partial \mu_{2}}{\partial x_{2}}>\frac{\partial \mu_{2}}{\partial x_{1}} .
$$

We recall that Theorem 1 and Proposition 5 assert that:

If Case 1 and (26) hold, then the positive steady state is unique and LES. Is it globally attractive?

If Case 3 [resp. Case 4] and (26) or (29) hold, then the positive steady state does not exist and $\mathcal{E}_{1}$ [resp. $\left.\mathcal{E}_{2}\right]$ is LES. Is it globally attractive?

If $\mu_{i}\left(S_{i n}, 0,0\right)<D, i=1,2$ then $\mathcal{E}_{1}$ and $\mathcal{E}_{2}$ do not exist and $\mathcal{E}_{0}$ is LES. Is it globally attractive?

If Case 2 holds, then $\mathcal{E}_{1}$ and $\mathcal{E}_{2}$ are both LES. Therefore, we cannot expect global attractivity of the boundary steady states. Note that if, in addition, (29) holds, the positive steady state is unique and is unstable.

The aim of this section is to give the global behavior of the above-mentioned steady states. Written in the variables $\left(z, x_{1}, x_{2}\right)$, the system (4) becomes

$$
\left\{\begin{array}{l}
\dot{z}=D\left(S_{i n}-z\right) \\
\dot{x}_{1}=\left[\mu_{1}\left(z-x_{1}-x_{2}, x_{1}, x_{2}\right)-D\right] x_{1} \\
\dot{x}_{2}=\left[\mu_{2}\left(z-x_{1}-x_{2}, x_{2}, x_{1}\right)-D\right] x_{2}
\end{array}\right.
$$

Let $\left(z(t), x_{1}(t), x_{2}(t)\right)$ be a solution of (30). From the first equation, we deduce that

$$
z(t)=S_{\text {in }}+\left(z(0)-S_{\text {in }}\right) e^{-D t} .
$$

Thus, (30) is equivalent to non-autonomous system of two differential equations

$$
\left\{\begin{array}{l}
\dot{x}_{1}=\left[\mu_{1}\left(S_{i n}+\left(z(0)-S_{i n}\right) e^{-D t}-x_{1}-x_{2}, x_{1}, x_{2}\right)-D\right] x_{1} \\
\dot{x}_{2}=\left[\mu_{2}\left(S_{i n}+\left(z(0)-S_{i n}\right) e^{-D t}-x_{1}-x_{2}, x_{2}, x_{1}\right)-D\right] x_{2}
\end{array}\right.
$$

This is an asymptotically autonomous differential system which converges toward the autonomous system

$$
\left\{\begin{array}{l}
\dot{x}_{1}=x_{1} f_{1}\left(x_{1}, x_{2}\right) \\
\dot{x}_{2}=x_{2} f_{2}\left(x_{1}, x_{2}\right)
\end{array}\right.
$$

where the functions $f_{1}$ and $f_{2}$ are defined by (19), with $D_{1}=D_{2}=D$, that is

$$
f_{1}\left(x_{1}, x_{2}\right):=\mu_{1}\left(S_{\text {in }}-x_{1}-x_{2}, x_{1}, x_{2}\right)-D, \quad f_{2}\left(x_{1}, x_{2}\right):=\mu_{2}\left(S_{\text {in }}-x_{1}-x_{2}, x_{2}, x_{1}\right)-D .
$$

The results of Thieme [47], permit to deduce the asymptotic behavior of the solution of (31) from the asymptotic behavior of the autonomous system (32) which is called the reduced model since it is simply the restriction of (31) to the invariant plane defined by $z(0)=S_{\text {in }}$ for which $z(t)=S_{\text {in }}$ for all $t \geqslant 0$. 


\subsection{Reduced model}

In this section, we study the reduced system (32). This system is defined on the set

$$
M=\left\{\left(x_{1}, x_{2}\right) \in \mathbb{R}_{+}^{2}: x_{1}+x_{2} \leqslant S_{\text {in }}\right\} .
$$

The boundary of $M$ satisfies the following properties for $i=1,2$ :

$$
\begin{aligned}
& \left(x_{1}+x_{2}\right)(\tau)=S_{i n} \quad \text { for some } \tau \geqslant 0 \Longrightarrow \dot{x}_{i}(\tau)=-D x_{i}(\tau) \leqslant 0 \text {, } \\
& x_{i}(\tau)=0 \quad \text { for some } \tau \geqslant 0 \quad \Longrightarrow \quad \dot{x}_{i}(\tau)=0 \text {. }
\end{aligned}
$$

Hence, the set $M$ is bounded and positively invariant, that is, the system (32) is dissipative. Beside the washout $E_{0}=(0,0)$ which always exists, the system (32) can have a steady state of the form $E_{1}=\left(\tilde{x}_{1}, 0\right)$, and a steady state of the form $E_{2}=\left(0, \tilde{x}_{2}\right)$, where $\tilde{x}_{i}$ is a solution, if it exists, of the equation

$$
\mu_{i}\left(S_{\text {in }}-x_{i}, x_{i}, 0\right)=D, \quad i=1,2 .
$$

The system can have also a positive steady state $E^{*}=\left(x_{1}^{*}, x_{2}^{*}\right)$ where $\left(x_{1}^{*}, x_{2}^{*}\right)$ is a solution, if it exists, of two equations $f_{i}\left(x_{1}, x_{2}\right)=0, i=1,2$. Notice that to the steady states of (32)

$$
E_{0}=(0,0), \quad E_{1}=\left(\tilde{x}_{1}, 0\right), \quad E_{2}=\left(0, \tilde{x}_{2}\right) \quad \text { and } \quad E^{*}=\left(x_{1}^{*}, x_{2}^{*}\right)
$$

correspond the steady states of (4), respectively,

$$
\mathcal{E}_{0}=\left(S_{i n}, 0,0\right), \quad \mathcal{E}_{1}=\left(S_{\text {in }}-\tilde{x}_{1}, \tilde{x}_{1}, 0\right), \quad \mathcal{E}_{2}=\left(S_{\text {in }}-\tilde{x}_{2}, 0, \tilde{x}_{2}\right) \quad \text { and } \quad \mathcal{E}^{*}=\left(S_{\text {in }}-x_{1}^{*}-x_{2}^{*}, x_{1}^{*}, x_{2}^{*}\right) .
$$

\section{Proposition 7.}

1. If Case 1 holds and the positive steady state $E^{*}$ is unique, then it is GAS for (32) in the interior of $M$.

2. If Case 3 holds and there is no positive steady state, then $E_{1}$ is GAS for (32) in the interior of $M$.

3. If Case 4 holds and there is no positive steady state, then $E_{2}$ is GAS for (32) in the interior of $M$.

4. $E_{0}$ is GAS for (32) in $M$ if and only if $\mu_{i}\left(S_{\text {in }}, 0,0\right)<D$.
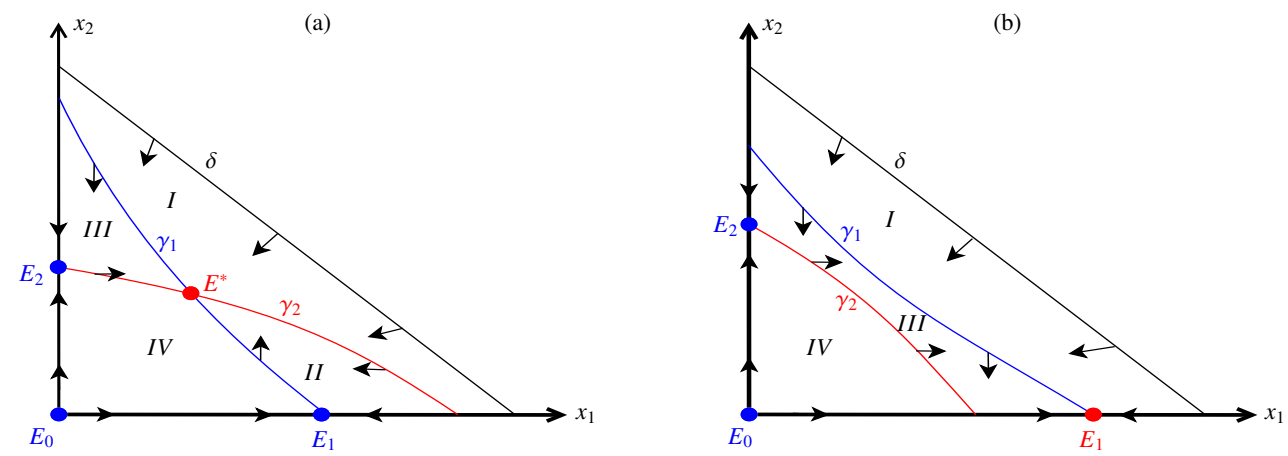

Figure 4: (a) Item 1 of Proposition 7, where $E^{*}$ is the global attractor. (b) Item 2 of Proposition 7, where $E_{1}$ is the global attractor.

Proof. It is known [37] that all solutions of the general model of two competing species, always converge to one of the steady states of the system. For complements and details, the reader is referred to [20], page 270. Since (32) is a particular case of the systems studied in [20,37], and since in each case of Proposition 7 the system has only one steady state which is LES, we deduce that this steady state is GAS in the interior of $M$. For the convenience of the reader, we give a direct proof, without using the general result in [20]. 
The curves $\gamma_{1}$ and $\gamma_{2}$ separate the interior of $M$ in a finite number of connected open sets of the following four types:

$$
\begin{array}{rlllll}
I: & \dot{x}_{1}<0, & \dot{x}_{2}<0, & I I: & \dot{x}_{1}<0, & \dot{x}_{2}>0, \\
I I I: & \dot{x}_{1}>0, & \dot{x}_{2}<0, & \text { IV }: & \dot{x}_{1}>0, & \dot{x}_{2}>0 .
\end{array}
$$

Consider a trajectory $\left(x_{1}(t), x_{2}(t)\right)$ starting at a positive initial condition $\left(x_{1}(0), x_{2}(0)\right)$.

If Case 1 holds and the positive steady state $E^{*}$ is unique, then the curves $\gamma_{1}$ and $\gamma_{2}$ have only one intersection and separate the interior of $M$ in four regions $I, I I, I I I$ and $I V$, see Figure 4(a). Assume first that $\left(x_{1}(0), x_{2}(0)\right) \in I I \cup I I I$. These regions are positively invariant. Since in $I I$ [resp. $I I I], x_{1}(t)$ is decreasing [resp. increasing] and $x_{2}(t)$ is increasing [resp. decreasing], the limits

$$
\lim _{t \rightarrow+\infty} x_{1}(t)=x_{1 \infty} \quad \text { and } \quad \lim _{t \rightarrow+\infty} x_{2}(t)=x_{2 \infty}
$$

Therefore, $\left(x_{1 \infty}, x_{2 \infty}\right)$ is a steady state of (32), which belongs to the closure $\overline{I I}$ or the closure $\overline{I I I}$. Since $E_{1}$ and $E^{*}$ [resp. $E_{2}$ and $E^{*}$ ] are the only steady states in $\overline{I I}$ [resp. $\overline{I I I}$ ] and since $E_{1}$ [resp. $E_{2}$ ] attracts only solutions with $x_{2}(0)=0$ [resp. $\left.x_{1}(0)=0\right]$, it follows that

$$
E^{*}=\left(x_{1 \infty}, x_{2 \infty}\right) .
$$

Assume now that $\left(x_{1}(0), x_{2}(0)\right) \in I$. If $\left(x_{1}(t), x_{2}(t)\right)$ remains in $I$ for all $t>0$ then $x_{1}(t)$ and $x_{2}(t)$ are positive decreasing functions. Thus, the limits (33) exist. Hence, $\left(x_{1 \infty}, x_{2 \infty}\right)$ is a steady state of (32) which belongs to the closure $\bar{I}$. Since $E^{*}$ is the only steady state in $\bar{I}$, we conclude that (34) holds. If $\left(x_{1}(t), x_{2}(t)\right)$ leaves the region $I$ and enters in the region $I I$ or $I I I$, then as shown previously it necessarily tends to $E^{*}$ and hence, (34) holds.

Assume now that $\left(x_{1}(0), x_{2}(0)\right) \in I V$. If $\left(x_{1}(t), x_{2}(t)\right)$ remains in $I V$ for all $t>0$ then $x_{1}(t)$ and $x_{2}(t)$ are bounded increasing functions. Thus, the limits (33) exist. Therefore, $\left(x_{1 \infty}, x_{2 \infty}\right)$ is a steady state of (32) which belongs to the closure $\overline{I V}$. Since $E_{0}, E_{1}, E_{2}$ and $E^{*}$ are the only steady states in $\overline{I V}$ and since $E_{0}$ is an unstable node, and $E_{1}$ [resp. $E_{2}$ ] is a saddle point that attracts only solutions with $x_{2}(0)=0$ [resp. $x_{1}(0)=0$ ], we conclude that (34) holds. If $\left(x_{1}(t), x_{2}(t)\right)$ leaves the region $I V$ and enters in the region $I I$ or $I I I$, then as shown previously it necessarily tends to $E^{*}$ and hence, (34) holds. Therefore, $E^{*}$ is GAS in the interior of $M$.

If Case 3 holds and there is no positive steady state, then the curves $\gamma_{1}$ and $\gamma_{2}$ do not intersect and separate the interior of $M$ in three regions $I, I I I$ and $I V$, see Figure 4(b). Assume first that $\left(x_{1}(0), x_{2}(0)\right) \in I I I$. This region is positively invariant. Since in $I I I, x_{1}(t)$ is increasing and $x_{2}(t)$ is decreasing, the limits (33) exist. Hence, $\left(x_{1 \infty}, x_{2 \infty}\right)$ is a steady state of (32) which belongs to the closure $\overline{I I I}$. Since $E_{1}$ and $E_{2}$ are the only steady states in $\overline{I I I}$ and since $E_{2}$ attracts only solutions with $x_{1}(0)=0$, we conclude that

$$
E_{1}=\left(x_{1 \infty}, x_{2 \infty}\right) .
$$

Assume now that $\left(x_{1}(0), x_{2}(0)\right) \in I$. If $\left(x_{1}(t), x_{2}(t)\right)$ remains in $I$ for all $t>0$ then $x_{1}(t)$ and $x_{2}(t)$ are positive decreasing functions. Hence the limits (33) exist. Therefore, $\left(x_{1 \infty}, x_{2 \infty}\right)$ is a steady state of (32) which belongs to the closure $\vec{I}$. Since $E_{1}$ is the only steady state in $\bar{I}$, we conclude that (35) holds. If $\left(x_{1}(t), x_{2}(t)\right)$ leaves the region $I$ and enters in the region $I I I$, then as shown previously it necessarily tends to $E_{1}$ and hence, (35) holds.

Assume now that $\left(x_{1}(0), x_{2}(0)\right) \in I V$. If $\left(x_{1}(t), x_{2}(t)\right)$ remains in $I V$ for all $t>0$ then $x_{1}(t)$ and $x_{2}(t)$ are bounded increasing functions. Thus, the limits (33) exist. Hence, $\left(x_{1 \infty}, x_{2 \infty}\right)$ is a steady state of (32) which belongs to the closure $\overline{I V}$. Since $E_{0}$ and $E_{2}$ are the only steady states in $\overline{I V}$ and since $E_{0}$ is an unstable node and $E_{2}$ attracts only solutions with $x_{1}(0)=0$, we conclude that the solution must leaves $I V$ and enters in $I I I$. As before, we see that (35) holds. Therefore, $E_{1}$ is GAS in the interior of $M$. The other cases can be proved using similar arguments.

\subsection{Global behavior}

Using Thieme's results [47], we deduce that the asymptotic behavior of the solution of three order system (4) is the same as the asymptotic behavior of second order system (32). The main result in this section can now be stated.

\section{Theorem 2.}

1. If Case 1 holds and the positive steady state $\mathcal{E}^{*}$ is unique, then it is GAS for (4) in the interior of $\Omega$.

2. If Case 3 holds and there is no positive steady state, then $\mathcal{E}_{1}$ is GAS for (4) in the interior of $\Omega$. 
3. If Case 4 holds and there is no positive steady state, then $\mathcal{E}_{2}$ is GAS for (4) in the interior of $\Omega$.

4. $\mathcal{E}_{0}$ is $G A S$ for (4) in $\Omega$ if and only if $\mu_{i}\left(S_{\text {in }}, 0,0\right)<D$.

Proof. The system (4) is equivalent to the non-autonomous system (31). This system is an asymptotically autonomous differential system which converges toward the autonomous system (32). According to Theorem 1, the study of phase portrait of reduced system (32) on $M$, shows that there exist only stable nodes, unstable nodes and saddle points. For any initial condition on the $x_{1}$ axis (resp. $x_{2}$ axis), the solution cannot converge to $E_{2}$ (resp. $E_{1}$ ) since $E_{0}$ is an unstable node. Hence, there is no trajectory joining two saddle points. Consequently, we can apply Thieme's results [47] and conclude that the asymptotic behavior of the solution of (31) is the same as the reduced system (32).

The existence, local and global stability of all steady states of (4) are summarized in Table 1.

\begin{tabular}{llll}
\hline \hline Steady state & Existence & Local stability & Global stability \\
\hline $\mathcal{E}^{*}$ & $(25)$ has a solution & $F_{1}^{\prime}\left(x_{1}^{*}\right) F_{2}^{\prime}\left(x_{2}^{*}\right)>1$ & Case 1 holds and (25) has a unique solution \\
$\mathcal{E}_{1}$ & $\mu_{1}\left(S_{i n}, 0,0\right)>D$ & $\bar{x}_{1}<\tilde{x}_{1}$ & Case 3 holds and (25) has no solution \\
$\mathcal{E}_{2}$ & $\mu_{2}\left(S_{i n}, 0,0\right)>D$ & $\bar{x}_{2}<\tilde{x}_{2}$ & Case 4 holds and (25) has no solution \\
$\mathcal{E}_{0}$ & Always exists & $\mu_{i}\left(S_{i n}, 0,0\right)<D, i=1,2$ & $\mu_{i}\left(S_{i n}, 0,0\right)<D, i=1,2$ \\
\hline \hline
\end{tabular}

Table 1: Conditions of existence and stability of steady states in system (4).

\section{Applications}

In this section, we apply our results to the particular case $\mu_{i}\left(S, x_{i}, x_{j}\right)=v_{i}\left(S, x_{i}+\alpha_{i} x_{j}\right)$ where the functions $v_{i}(S, x)$ satisfy the following properties:

(P1) $v_{i}(0, x)=0$ and $v_{i}(S, x)>0$ for all $S>0$ and $x \geqslant 0$.

(P2) $\frac{\partial v_{i}}{\partial S}(S, x)>0$ and $\frac{\partial v_{i}}{\partial x}(S, x)<0$ for all $S>0$ and $x \geqslant 0$.

Since

$$
\frac{\partial \mu_{i}}{\partial x_{i}}=\frac{\partial v_{i}}{\partial x}, \quad \frac{\partial \mu_{i}}{\partial x_{j}}=\alpha_{i} \frac{\partial v_{i}}{\partial x},
$$

we have $\frac{\partial \mu_{i}}{\partial x_{i}}<0$ and $\frac{\partial \mu_{i}}{\partial x_{j}}<0$. Therefore, the growth functions $\mu_{i}$ satisfy conditions (H1) and (H2) so that our results apply to the following system:

$$
\left\{\begin{array}{l}
\dot{S}=D\left(S_{i n}-S\right)-v_{1}\left(S, x_{1}+\alpha_{1} x_{2}\right) x_{1}-v_{2}\left(S, x_{2}+\alpha_{2} x_{1}\right) x_{2} \\
\dot{x}_{1}=\left[v_{1}\left(S, x_{1}+\alpha_{1} x_{2}\right)-D_{1}\right] x_{1} \\
\dot{x}_{2}=\left[v_{2}\left(S, x_{2}+\alpha_{2} x_{1}\right)-D_{2}\right] x_{2} .
\end{array}\right.
$$

Notice that in (H2) we assumed only that the partial derivatives $\frac{\partial \mu_{i}}{\partial x_{i}} \leqslant 0$ and $\frac{\partial \mu_{i}}{\partial x_{j}} \leqslant 0$ : the case where some of these partial derivatives are zeros was not excluded. However, if all derivatives are zeros, then both intra- and interspecific competition is excluded, so that, no interesting phenomenon can occur. In the particular case considered in this section, if $\frac{\partial v_{i}}{\partial x}=0$ then $\frac{\partial \mu_{i}}{\partial x_{i}}=0$ and $\frac{\partial \mu_{i}}{\partial x_{j}}=0$, that is, there is no intra- nor interspecific competition. This is the reason why we used a strict inequality in $(\mathrm{P} 2)$. 


\subsection{Intra- and interspecific competition}

In this section, we consider the case of (37) where $\alpha_{i} \geqslant 0$, that is to say, both intra- and interspecific competition can occur. The case $\alpha_{1}=\alpha_{2}=0$ for which only intraspecific competition occurs will be considered in Section 5.2. The necessary and sufficient conditions (13) of existence of the boundary steady state $\mathcal{E}_{i}$ become

$$
v_{i}\left(S_{i n}, 0\right)>D_{i}, \quad i=1,2 .
$$

These two conditions are necessary for the existence of positive steady states. To have a better understanding of the existence of positive steady states, we must consider the relative positions of $\tilde{x}_{i}$ and $\bar{x}_{i}, i=1,2$ which play a major role in the distinctions between Cases 1, 2, 3 and 4 defined by (23) and (24).

We want to study how the relative positions of $\tilde{x}_{i}$ and $\bar{x}_{i}$ depend on the parameters $\alpha_{1}$ and $\alpha_{2}$. Recall that $\tilde{x}_{i}$ and $\bar{x}_{i}$ are the solutions of (11) and (22) respectively, which become

$$
v_{i}\left(S_{i n}-\frac{D_{i}}{D} \tilde{x}_{i}, \tilde{x}_{i}\right)=D_{i}, \quad v_{j}\left(S_{i n}-\frac{D_{i}}{D} \bar{x}_{i}, \alpha_{j} \bar{x}_{i}\right)=D_{j} .
$$

Therefore, $\tilde{x}_{i}$ does not depend on the parameters $\alpha_{i}$ and $\bar{x}_{i}$ depends on these parameters. Thus, we denote it by $\bar{x}_{i}\left(\alpha_{j}\right)$ to emphasize the dependence of $\bar{x}_{i}$ on the parameter $\alpha_{j}, j \neq i$. Each value of the parameter $\alpha_{1}$ or $\alpha_{2}$ for which $\bar{x}_{1}\left(\alpha_{2}\right)=\tilde{x}_{1}$ or $\bar{x}_{2}\left(\alpha_{1}\right)=\tilde{x}_{2}$ plays a role in the discussion. The next lemma shows the existence of critical value $\alpha_{j}=\alpha_{j}^{c}$ such that $\bar{x}_{i}\left(\alpha_{j}\right)=\tilde{x}_{i}$ for $i=1,2, j=1,2, i \neq j$.
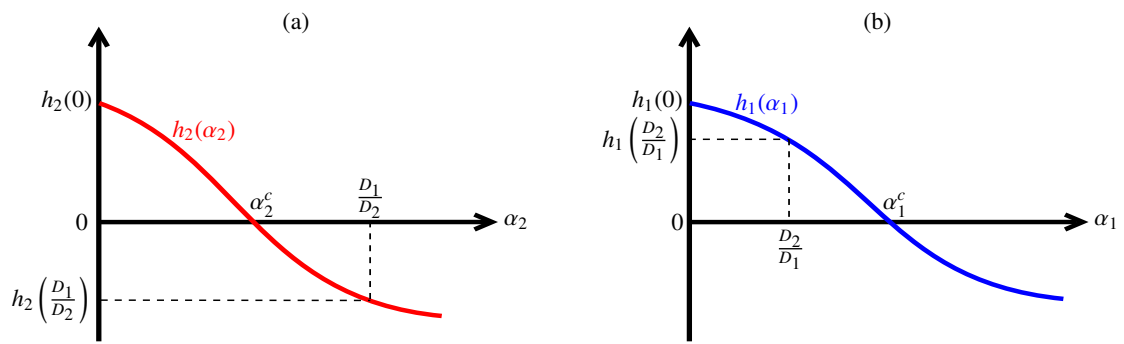

Figure 5: The case $D_{2} \tilde{x}_{2} \leqslant D_{1} \tilde{x}_{1}$ : Existence of two critical values (a) $\alpha_{2}^{c} \leqslant \frac{D_{1}}{D_{2}}$ and (b) $\alpha_{1}^{c} \geqslant \frac{D_{2}}{D_{1}}$.

Lemma 3. If $\bar{x}_{i}(0)<\tilde{x}_{i}, i=1,2$ then the equation $\bar{x}_{i}\left(\alpha_{j}\right)=\tilde{x}_{i}$ has no solution. If $\bar{x}_{i}(0) \geqslant \tilde{x}_{i}, i=1,2$ there exist two cases that must be distinguished: $D_{2} \tilde{x}_{2} \leqslant D_{1} \tilde{x}_{1}$ and $D_{1} \tilde{x}_{1}<D_{2} \tilde{x}_{2}$.

1. Case $D_{2} \tilde{x}_{2} \leqslant D_{1} \tilde{x}_{1}$ : There exists a unique critical value $\alpha_{2}^{c} \leqslant D_{1} / D_{2}$ such that $\bar{x}_{1}\left(\alpha_{2}\right) \geqslant \tilde{x}_{1}$ if and only if $\alpha_{2} \leqslant \alpha_{2}^{c}$. If $v_{1}\left(S_{i n}-\frac{D_{2}}{D} \tilde{x}_{2},+\infty\right)<D_{1}$, then there exists a unique critical value $\alpha_{1}^{c} \geqslant D_{2} / D_{1}$ such that $\bar{x}_{2}\left(\alpha_{1}\right) \geqslant \tilde{x}_{2}$ if and only if $\alpha_{1} \leqslant \alpha_{1}^{c}$. If $v_{1}\left(S_{\text {in }}-\frac{D_{2}}{D} \tilde{x}_{2},+\infty\right) \geqslant D_{1}$, then $\bar{x}_{2}\left(\alpha_{1}\right)>\tilde{x}_{2}$ for all $\alpha_{1} \geqslant 0$ and we let $\alpha_{1}^{c}=+\infty$.

2. Case $D_{1} \tilde{x}_{1}<D_{2} \tilde{x}_{2}$ : There exists a unique critical value $\alpha_{1}^{c}<D_{2} / D_{1}$ such that $\bar{x}_{2}\left(\alpha_{1}\right) \geqslant \tilde{x}_{2}$ if and only if $\alpha_{1} \leqslant \alpha_{1}^{c}$. If $v_{2}\left(S_{\text {in }}-\frac{D_{1}}{D} \tilde{x}_{1},+\infty\right)<D_{2}$, then there exists a unique critical value $\alpha_{2}^{c}>D_{1} / D_{2}$ such that $\bar{x}_{1}\left(\alpha_{2}\right) \geqslant \tilde{x}_{1}$ if and only if $\alpha_{2} \leqslant \alpha_{2}^{c}$. If $v_{2}\left(S_{\text {in }}-\frac{D_{1}}{D} \tilde{x}_{1},+\infty\right) \geqslant D_{2}$, then $\bar{x}_{1}\left(\alpha_{2}\right)>\tilde{x}_{1}$ for all $\alpha_{2} \geqslant 0$ and we let $\alpha_{2}^{c}=+\infty$.

Proof. Since $\bar{x}_{i}\left(\alpha_{j}\right)$ is given by the solution of (39), we note that $\bar{x}_{i}\left(\alpha_{j}\right)=\tilde{x}_{i}$ if and only if $h_{j}\left(\alpha_{j}\right)=0$ where

$$
h_{j}\left(\alpha_{j}\right)=v_{j}\left(S_{i n}-\frac{D_{i}}{D} \tilde{x}_{i}, \alpha_{j} \tilde{x}_{i}\right)-D_{j}=0, \quad \text { for } \quad i=1,2, j=1,2, \quad i \neq j .
$$

If $\bar{x}_{i}(0)<\tilde{x}_{i}, i=1,2$ then $S_{i n}-\frac{D_{i}}{D} \tilde{x}_{i}<S_{i n}-\frac{D_{i}}{D} \bar{x}_{i}(0)$. Since $\bar{x}_{i}\left(\alpha_{j}\right)$ is given by the solution of (39), it follows that

$$
h_{j}(0)=v_{j}\left(S_{i n}-\frac{D_{i}}{D} \tilde{x}_{i}, 0\right)-D_{j}<v_{j}\left(S_{i n}-\frac{D_{i}}{D} \bar{x}_{i}(0), 0\right)-D_{j}=0
$$


because the function $v_{j}(\cdot, 0)$ is increasing. Furthermore, for all $\alpha_{j}>0$, one has

$$
h_{j}^{\prime}\left(\alpha_{j}\right)=\tilde{x}_{i} \frac{\partial v_{j}}{\partial x}<0, \quad i=1,2, j=1,2, \quad i \neq j
$$

Therefore, the equation $h_{j}\left(\alpha_{j}\right)=0$ has no solution. If $\bar{x}_{i}(0) \geqslant \tilde{x}_{i}, i=1,2$ then $h_{i}(0) \geqslant 0$. If $D_{2} \tilde{x}_{2} \leqslant D_{1} \tilde{x}_{1}$ one has

$$
h_{2}\left(\frac{D_{1}}{D_{2}}\right)=\psi_{2}\left(\frac{D_{1}}{D_{2}} \tilde{x}_{1}\right) \leqslant \psi_{2}\left(\tilde{x}_{2}\right)=0 .
$$

Since $h_{2}(0) \geqslant 0$ and the function $h_{2}(\cdot)$ is decreasing, we deduce that there exists a unique solution $\alpha_{2}^{c} \in\left[0, D_{1} / D_{2}\right]$ such that $h_{2}\left(\alpha_{2}^{c}\right)=0$ (see Figure 5(a)). Let $\Phi_{i}(x)$ be the function defined by

$$
\Phi_{i}(x):=v_{i}\left(S_{i n}-\frac{D_{j}}{D} x, \alpha_{i} x\right)-D_{i} .
$$

One has $\alpha_{2}<\alpha_{2}^{c}$ if and only if $h_{2}\left(\alpha_{2}\right)>0$. This inequality is equivalent to

$$
v_{2}\left(S_{\text {in }}-\frac{D_{1}}{D} \tilde{x}_{1}, \alpha_{2} \tilde{x}_{1}\right)-D_{2}>v_{2}\left(S_{\text {in }}-\frac{D_{1}}{D} \bar{x}_{1}\left(\alpha_{2}\right), \alpha_{2} \bar{x}_{1}\left(\alpha_{2}\right)\right)-D_{2}=0
$$

or (equivalently) $\Phi_{2}\left(\tilde{x}_{1}\right)>\Phi_{2}\left(\bar{x}_{1}\left(\alpha_{2}\right)\right)$. Since the function $\Phi_{2}(\cdot)$ is decreasing, it follows that $\tilde{x}_{1}<\bar{x}_{1}\left(\alpha_{2}\right)$ if and only if $\alpha_{2}<\alpha_{2}^{c}$. We have also

$$
h_{1}\left(\frac{D_{2}}{D_{1}}\right)=\psi_{1}\left(\frac{D_{2}}{D_{1}} \tilde{x}_{2}\right) \geqslant \psi_{1}\left(\tilde{x}_{1}\right)=0 .
$$

If $v_{1}\left(S_{\text {in }}-\frac{D_{2}}{D} \tilde{x}_{2},+\infty\right)<D_{1}$, which means that

$$
\lim _{\alpha_{1} \longrightarrow+\infty} h_{1}\left(\alpha_{1}\right)<0
$$

then there exists a unique solution $\alpha_{1}^{c} \in\left[D_{2} / D_{1},+\infty\right)$ such that $h_{1}\left(\alpha_{1}^{c}\right)=0$, because the function $h_{1}(\cdot)$ is decreasing (see Figure 5(b)). Similarly, one has $\alpha_{1}<\alpha_{1}^{c}$ if and only if $h_{1}\left(\alpha_{1}\right)>0$ which is equivalent to $\Phi_{1}\left(\tilde{x}_{2}\right)>\Phi_{1}\left(\bar{x}_{2}\left(\alpha_{1}\right)\right)$. Since the function $\Phi_{1}(\cdot)$ is decreasing, it follows that $\tilde{x}_{2}<\bar{x}_{2}\left(\alpha_{1}\right)$ if and only if $\alpha_{1}<\alpha_{1}^{c}$. If $v_{1}\left(S_{\text {in }}-\frac{D_{2}}{D} \tilde{x}_{2},+\infty\right) \geqslant D_{1}$, we deduce that $h_{1}\left(\alpha_{1}\right)>0$ for all $\alpha_{1} \geqslant 0$. Similarly, we conclude that $\tilde{x}_{2}<\bar{x}_{2}\left(\alpha_{1}\right)$ for all $\alpha_{1} \geqslant 0$. The case $D_{1} \tilde{x}_{1}<D_{2} \tilde{x}_{2}$ can be proved in the same manner.

As a corollary of Theorem 1, we obtain the following result:

Proposition 8. Assume that (P1), (P2) and (38) hold.

1. Blue positive steady states are unstable. If $\alpha_{1}<1$ and $\alpha_{2}<1$ hold, or $D_{1}=D_{2}=D$, then red positive steady states are LES, that is to say, positive steady states are alternatively unstable and LES.

2. If $\alpha_{1}<\alpha_{1}^{c}$ and $\alpha_{2}<\alpha_{2}^{c}$ hold, then Case 1 holds, so that the system can have generically an odd number of positive steady states, while $\mathcal{E}_{1}$ and $\mathcal{E}_{2}$ are unstable. The positive steady states at the left-hand end and right-hand end are red. If, in addition, $\alpha_{1}<D_{2} / D_{1}$ and $\alpha_{2}<D_{1} / D_{2}$ hold, then the positive steady state is unique.

3. If $\alpha_{1}>\alpha_{1}^{c}$ and $\alpha_{2}>\alpha_{2}^{c}$ hold, then Case 2 holds, so that the system can have generically an odd number of positive steady states, while $\mathcal{E}_{1}$ and $\mathcal{E}_{2}$ are LES. The positive steady states at the left-hand end and right-hand end are blue. If, in addition, $\alpha_{1}>D_{2} / D_{1}$ and $\alpha_{2}>D_{1} / D_{2}$ hold, then $\mathcal{E}^{*}$ is unique and unstable.

4. If $\alpha_{1}<\alpha_{1}^{c}$ and $\alpha_{2}>\alpha_{2}^{c}$ [resp. $\alpha_{1}>\alpha_{1}^{c}$ and $\alpha_{2}<\alpha_{2}^{c}$ ] hold, then Case 3 [resp. Case 4] holds, so that generically the system has no positive steady state or an even number of positive steady states where $\mathcal{E}_{1}$ is LES [resp. unstable] and $\mathcal{E}_{2}$ is unstable [resp. LES]. The positive steady state at the right-hand [resp. left-hand] end, if it exists, is blue. If, in addition, $\alpha_{1}<D_{2} / D_{1}$ and $\alpha_{2}<D_{1} / D_{2}$ hold or $\alpha_{1}>D_{2} / D_{1}$ and $\alpha_{2}>D_{1} / D_{2}$ hold, then the system has no positive steady state. 
Proof. The formulas (36) show that: the conditions (26) are equivalent to $\alpha_{1}<1$ and $\alpha_{2}<1$; the conditions (27) are equivalent to $\alpha_{1}<D_{2} / D_{1}$ and $\alpha_{2}<D_{1} / D_{2}$; the conditions (28) are equivalent to $\alpha_{1}>D_{2} / D_{1}$ and $\alpha_{2}>D_{1} / D_{2}$.

Moreover, Lemma 3 shows that: Case 1 is equivalent to $\alpha_{1}<\alpha_{1}^{c}$ and $\alpha_{2}<\alpha_{2}^{c}$; Case 2 is equivalent to $\alpha_{1}>\alpha_{1}^{c}$ and $\alpha_{2}>\alpha_{2}^{c}$; Case 3 is equivalent to $\alpha_{1}<\alpha_{1}^{c}$ and $\alpha_{2}>\alpha_{2}^{c}$; Case 4 is equivalent to $\alpha_{1}>\alpha_{1}^{c}$ and $\alpha_{2}<\alpha_{2}^{c}$.

Therefore, Theorem 1 applies and gives the result.

For more clarity, let us summarize in Tables the various cases which were encountered in Proposition 8 . Without loss of generality, we can assume that $D_{1}<D_{2}$. If $D_{2} \tilde{x}_{2}<D_{1} \tilde{x}_{1}$ then $\alpha_{2}^{c}<D_{1} / D_{2}<1<D_{2} / D_{1}<\alpha_{1}^{c}$, and the results of Proposition 8 are given in Table 2.

\begin{tabular}{c||c|c|c|c}
$D_{2} \tilde{x}_{2}<D_{1} \tilde{x}_{1}$ & $0 \leqslant \alpha_{1}<1$ & $1<\alpha_{1}<D_{2} / D_{1}$ & $D_{2} / D_{1}<\alpha_{1}<\alpha_{1}^{c}$ & $\alpha_{1}^{c}<\alpha_{1}$ \\
\hline \hline $0 \leq \alpha_{2}<\alpha_{2}^{c}$ & Case 1.aS & Case 1.a & Case 1 & Case 4 \\
$\alpha_{2}^{c}<\alpha_{2}<D_{1} / D_{2}$ & Case 3.a & Case 3.a & Case 3 & Case 2 \\
$D_{1} / D_{2}<\alpha_{2}<1$ & Case 3S & Case 3 & Case 3.a & Case 2.a \\
$1<\alpha_{2}$ & Case 3 & Case 3 & Case 3.a & Case 2.a
\end{tabular}

Table 2: Existence and stability of steady states of (37) according to $\alpha_{i}, i=1,2$, when $D_{2} \tilde{x}_{2}<D_{1} \tilde{x}_{1}$. The suffix $\mathrm{S}$ added to a Case means that red positive steady states are stable: Case 1.aS means that the unique positive steady state is stable; Case $3 \mathrm{~S}$ means that all possible red positive steady states of Case 3 are stable.

If $D_{2} \tilde{x}_{2}>D_{1} \tilde{x}_{1}$ then four cases must be distinguished:

$$
\begin{array}{ll}
D_{1} / D_{2}<\alpha_{2}^{c}<1 \text { and } 1<\alpha_{1}^{c}<D_{2} / D_{1}, \quad D_{1} / D_{2}<1<\alpha_{2}^{c} \text { and } 1<\alpha_{1}^{c}<D_{2} / D_{1}, \\
D_{1} / D_{2}<\alpha_{2}^{c}<1 \text { and } \alpha_{1}^{c}<1<D_{2} / D_{1}, & D_{1} / D_{2}<1<\alpha_{2}^{c} \text { and } \alpha_{1}^{c}<1<D_{2} / D_{1} .
\end{array}
$$

We consider only the last case, the other ones can be discussed similarly. The results of Proposition 8 are given in Table 3.

\begin{tabular}{c||c|c|c|c}
$D_{2} \tilde{x}_{2}>D_{1} \tilde{x}_{1}$ & $0 \leqslant \alpha_{1}<\alpha_{1}^{c}$ & $\alpha_{1}^{c}<\alpha_{1}<1$ & $1<\alpha_{1}<D_{2} / D_{1}$ & $D_{2} / D_{1}<\alpha_{1}$ \\
\hline \hline $0 \leq \alpha_{2}<D_{1} / D_{2}$ & Case 1.aS & Case 4.a & Case 4.a & Case 4 \\
$D_{1} / D_{2}<\alpha_{2}<1$ & Case 1S & Case 4S & Case 4 & Case 4.a \\
$1<\alpha_{2}<\alpha_{2}^{c}$ & Case 1 & Case 4 & Case 4 & Case 4.a \\
$\alpha_{2}^{c}<\alpha_{2}$ & Case 3 & Case 2 & Case 2 & Case 2.a
\end{tabular}

Table 3: Existence and stability of steady states of (37) according to $\alpha_{i}, i=1,2$, when $D_{2} \tilde{x}_{2}>D_{1} \tilde{x}_{1}$. The suffix $\mathrm{S}$ added to a Case means that red positive steady states are stable.

When the dilutions rates are equal $D_{1}=D_{2}=D$, we have only two cases to consider: If $\tilde{x}_{2}<\tilde{x}_{1}$, then $\alpha_{2}^{c}<1<\alpha_{1}^{c}$, so that the results of Proposition 8 are given in Table 4 .

\begin{tabular}{c||c|c|c}
$\tilde{x}_{2}<\tilde{x}_{1}$ & $0 \leqslant \alpha_{1}<1$ & $1<\alpha_{1}<\alpha_{1}^{c}$ & $\alpha_{1}^{c}<\alpha_{1}$ \\
\hline \hline $0 \leq \alpha_{2}<\alpha_{2}^{c}$ & Case 1.aS & Case 1S & Case 4S \\
$\alpha_{2}^{c}<\alpha_{2}<1$ & Case 3.a & Case 3S & Case 2S \\
$1<\alpha_{2}$ & Case 3S & Case 3.a & Case 2.a
\end{tabular}

Table 4: Existence and stability of steady states of (37) according to $\alpha_{i}, i=1,2$, when $D_{1}=D_{2}=D$ and $\tilde{x}_{2}<\tilde{x}_{1}$. The suffix S added to a Case means that red positive steady states are stable.

If $\tilde{x}_{2}>\tilde{x}_{1}$, then $\alpha_{1}^{c}<1<\alpha_{2}^{c}$, so that the results of Proposition 8 are given in Table 5 .

\subsection{No interspecific competition : the steady-state characteristic}

In this section, we compare our results with those obtained by Lobry et al. [34, 35] in the case of (37) where only the intraspecific competition is taken into account, that is to say $\alpha_{1}=\alpha_{2}=0$. Following these authors, we use the steady-state characteristic to describe the steady states of the system. We begin by recalling the definition of the 


\begin{tabular}{c|c|c|c}
$\tilde{x}_{2}>\tilde{x}_{1}$ & $0 \leqslant \alpha_{1}<\alpha_{1}^{c}$ & $\alpha_{1}^{c}<\alpha_{1}<1$ & $1<\alpha_{1}$ \\
\hline \hline $0 \leq \alpha_{2}<1$ & Case 1.aS & Case 4.a & Case 4S \\
$1<\alpha_{2}<\alpha_{2}^{c}$ & Case 1S & Case 4S & Case 4.a \\
$\alpha_{2}^{c}<\alpha_{2}$ & Case 3S & Case 2S & Case 2.a
\end{tabular}

Table 5: Existence and stability of steady states of (37) according to $\alpha_{i}, i=1,2$, when $D_{1}=D_{2}=D$ and $\tilde{x}_{2}>\tilde{x}_{1}$. The suffix $\mathrm{S}$ added to a Case means that red positive steady states are stable.

break-even concentrations. By (P2), the function $S \mapsto v_{i}(S, 0)$ is increasing. Hence, if $D_{i}<v_{i}(+\infty, 0)$ then there exists a unique value $\lambda_{i}$ (called the break-even concentration) such that

$$
v_{i}\left(\lambda_{i}, 0\right)=D_{i}, \quad i=1,2 .
$$

If $D_{i} \geqslant v_{i}(+\infty, 0)$, we let $\lambda_{i}=+\infty$. In order to determine the condition of existence of steady states, we consider the function $S \mapsto x=M_{i}(S)$ defined by the equation $v_{i}(S, x)=D_{i}$. More precisely, by (P2), the function $x \mapsto v_{i}(S, x)$ is decreasing. Let $S \geqslant \lambda_{i}$. Then $v_{i}(S, 0) \geqslant D_{i}$. If $v_{i}(S,+\infty)<D_{i}$ then there exists a unique $x=M_{i}(S) \geqslant 0$ such that

$$
v_{i}(S, x)=D_{i} \quad \Longleftrightarrow \quad x=M_{i}(S), \text { for } S \geqslant \lambda_{i}
$$

Notice that the function

$$
M_{i}: I_{i} \longrightarrow[0+\infty), \quad I_{i}=\left[\lambda_{i}, \lambda_{i}^{\prime}\right)
$$

is defined for $S \in\left[\lambda_{i}, \lambda_{i}^{\prime}\right)$ where $\lambda_{i}^{\prime}>\lambda_{i}$ is defined as follows: if equation $D_{i}=v_{i}(S,+\infty)$ has a solution then $\lambda_{i}^{\prime}$ is that solution, that is $v_{i}\left(\lambda_{i}^{\prime},+\infty\right)=D_{i}$ and if $D_{i}>v_{i}(S,+\infty)$ for all $S>0$ then $\lambda_{i}^{\prime}=+\infty$. Notice that the function $M_{i}(\cdot)$ is increasing and satisfies $M_{i}\left(\lambda_{i}\right)=0$ and $M_{i}\left(\lambda_{i}^{\prime}\right)=+\infty$. Let $\Gamma_{i}: I_{i} \rightarrow[0+\infty), i=1,2,3$ defined by

$$
\Gamma_{1}(S)=\frac{D_{1}}{D} M_{1}(S), \quad \Gamma_{2}(S)=\frac{D_{2}}{D} M_{2}(S), \quad \Gamma_{3}(S)=\frac{D_{1}}{D} M_{1}(S)+\frac{D_{2}}{D} M_{2}(S) .
$$

The graphs of these functions are denoted by $C_{i}, i=1,2,3$. Let us denote by $C_{0}$ the positive semi $S$-axis.

Definition 2. The steady-state characteristic is $C=C_{0} \cup C_{1} \cup C_{2} \cup C_{3}$, the reunion of the curves $C_{i}, i=0,1,2,3$.
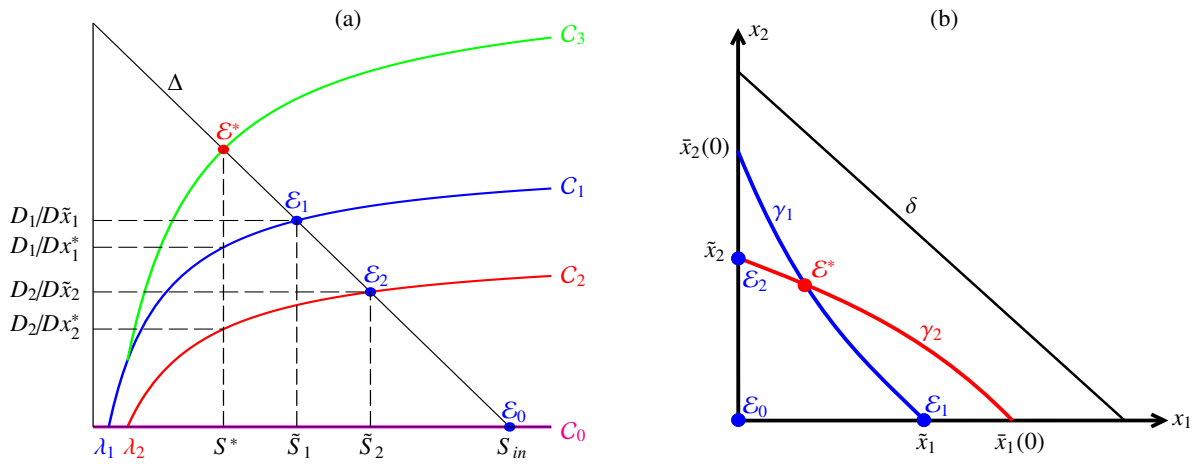

Figure 6: The case $\alpha_{1}=\alpha_{2}=0$ : (a) the steady-state characteristic, (b) condition of existence and uniqueness of the positive steady state.

The name of this set follows from the property that the values of $S$ at steady states are obtained simply at the intersection of the steady-state characteristic $C$ with the graph $\Delta$ of the affine function $S \mapsto S_{\text {in }}-S$ (see Figure 6(a)). More precisely, we have the following result:

Proposition 9. The intersection $S=S_{\text {in }}$ of $\Delta$ with $C_{0}$ is the $S$-component of the washout $\mathcal{E}_{0}=\left(S_{\text {in }}, 0,0\right)$.

The intersection $S=\tilde{S}_{i}$ of $\Delta$ with $C_{i}, i=1,2$ is the $S$-component of the boundary steady state $\mathcal{E}_{i}$. The $x_{i}$ component of this steady state is given by

$$
\tilde{x}_{i}=M_{i}\left(\tilde{S}_{i}\right) .
$$

This intersection exists if and only if $S_{\text {in }}>\lambda_{i}$ which is equivalent to (38). It is unique if it exists. 
The intersection $S=S^{*}$ of $\Delta$ with $C_{3}$, is the $S$-component of the positive steady state $\mathcal{E}^{*}$. The $x_{1}$ and $x_{2}$ components of this steady state are given by

$$
x_{1}^{*}=M_{1}\left(S^{*}\right), \quad x_{2}^{*}=M_{2}\left(S^{*}\right) .
$$

This intersection exists if and only if

$$
S_{\text {in }}-\tilde{\lambda}>\frac{D_{1}}{D} M_{1}(\tilde{\lambda})+\frac{D_{2}}{D} M_{2}(\tilde{\lambda})
$$

where $\tilde{\lambda}=\max \left(\lambda_{1}, \lambda_{2}\right)$. It is unique if it exists.

Proof. In the particular case $\mu_{i}\left(S, x_{i}, x_{j}\right)=v_{i}\left(S, x_{i}\right)$, the equations $(8,9)$ giving a boundary steady state $\mathcal{E}_{i}, i=1,2$ become

$$
D\left(S_{\text {in }}-\tilde{S}_{i}\right)=D_{i} \tilde{x}_{i}, \quad v_{i}\left(\tilde{S}_{i}, \tilde{x}_{i}\right)=D_{i} .
$$

Therefore, using the notation (41), we must have (42) and $S=\tilde{S}_{i}$ is a solution of

$$
S_{\text {in }}-S=\frac{D_{i}}{D} M_{i}(S)
$$

Thus, $\tilde{S}_{i}$ is the $S$-component of the intersection of $\Delta$ with $C_{i}, i=1,2$. Since $\tilde{S}_{i}>\lambda_{i}, \mathcal{E}_{i}$ exists if and only if $S_{i n}>\lambda_{i}$, that is to say (38) holds. Since the function $\Gamma_{i}$ is increasing, this intersection is unique if it exists.

Similarly, the equations $(14,15,16)$ giving a positive steady state $\mathcal{E}^{*}$, become

$$
D\left(S_{\text {in }}-S\right)=D_{1} x_{1}+D_{2} x_{2}, \quad v_{1}\left(S, x_{1}\right)=D_{1}, \quad v_{2}\left(S, x_{2}\right)=D_{2}
$$

Therefore, using the notation (41), we must have (43) and $S=S^{*}$ must be a solution of

$$
S_{\text {in }}-S=\frac{D_{1}}{D} M_{1}(S)+\frac{D_{2}}{D} M_{2}(S)
$$

Thus, $S^{*}$ is the $S$-component of the intersection of $\Delta$ with $C_{3}$. Since $S^{*}>\tilde{\lambda}=\max \left(\lambda_{1}, \lambda_{2}\right), \mathcal{E}^{*}$ exists if and only if (44) holds. Since the function $\Gamma_{3}$ is increasing, this intersection is unique if it exists.

The formulas (42) and (43) show that the component $\tilde{x}_{i}$ of $\mathcal{E}_{i}$ and the components $x_{1}^{*}$ and $x_{2}^{*}$ of $\mathcal{E}^{*}$ are given as in Figure 6(a). The condition (44) of existence of the positive steady state $\mathcal{E}^{*}$ was obtained by Lobry et al. [34, 35] in the particular case $D_{1}=D_{2}=D$. Let us show that this condition is equivalent to Case 1 considered in the present paper, that is to say, see Figure 6(b):

$$
\bar{x}_{1}(0)>\tilde{x}_{1} \quad \text { and } \quad \bar{x}_{2}(0)>\tilde{x}_{2} .
$$

Recall that $\tilde{x}_{i}$ and $\bar{x}_{i}(0), i=1,2$ are the solutions of (39). Hence we have

$$
v_{i}\left(S_{i n}-\frac{D_{i}}{D} \tilde{x}_{i}, \tilde{x}_{i}\right)=D_{i}, \quad v_{j}\left(S_{\text {in }}-\frac{D_{i}}{D} \bar{x}_{i}(0), 0\right)=D_{j}, \quad i=1,2, j=1,2, \quad i \neq j .
$$

Notice that according to Proposition 8, if Case 1 holds, the positive steady state is unique and LES, since in the particular case of this section, $\alpha_{1}=0$ and $\alpha_{2}=0$, so that conditions $\alpha_{1}<1, \alpha_{2}<1, \alpha_{1}<D_{2} / D_{1}$ and $\alpha_{2}<D_{1} / D_{2}$ are satisfied.

Lemma 4. The conditions (44) and (45) are both equivalent to

$$
v_{1}\left(\lambda_{2}, \frac{D}{D_{1}}\left(S_{\text {in }}-\lambda_{2}\right)\right)<D_{1} \quad \text { and } \quad v_{2}\left(\lambda_{1}, \frac{D}{D_{2}}\left(S_{\text {in }}-\lambda_{1}\right)\right)<D_{2} .
$$

Proof. Without loss of generality, one may assume that $\lambda_{1}<\lambda_{2}$. So, the condition (44) is equivalent to

$$
S_{\text {in }}-\lambda_{1}>S_{\text {in }}-\lambda_{2}>\frac{D_{1}}{D} M_{1}\left(\lambda_{2}\right)>0
$$


Since the functions $x \mapsto v_{i}\left(\lambda_{j}, x\right)$ are decreasing and by definition (41) of the function $M_{1}$, condition (48) is equivalent to

$$
\left\{\begin{array}{l}
v_{1}\left(\lambda_{2}, \frac{D}{D_{1}}\left(S_{\text {in }}-\lambda_{2}\right)\right)-D_{1}<v_{1}\left(\lambda_{2}, M_{1}\left(\lambda_{2}\right)\right)-D_{1}=0 \\
v_{2}\left(\lambda_{1}, \frac{D}{D_{2}}\left(S_{\text {in }}-\lambda_{1}\right)\right)-D_{2}<v_{2}\left(\lambda_{1}, 0\right)-D_{2}<v_{2}\left(\lambda_{2}, 0\right)-D_{2}=0
\end{array}\right.
$$

which is the same as (47). For the proof of the equivalence of (45) and (47), we recall that the function $\psi_{i}\left(x_{i}\right)$ defined by (12) which becomes now

$$
\psi_{i}\left(x_{i}\right)=v_{i}\left(S_{\text {in }}-\frac{D_{i}}{D} x_{i}, x_{i}\right)-D_{i}, \quad i=1,2
$$

vanishes at $\tilde{x}_{i}$ and is decreasing. As $\bar{x}_{i}(0)$ is the unique solution of (46), it follows form definition (40) of the break-even concentration $\lambda_{i}$ that $\bar{x}_{i}(0)=\frac{D}{D_{i}}\left(S_{i n}-\lambda_{j}\right)$. Therefore, condition (45) is equivalent to

$$
v_{2}\left(\lambda_{1}, \frac{D}{D_{2}}\left(S_{\text {in }}-\lambda_{1}\right)\right)-D_{2}=\psi_{2}\left(\bar{x}_{2}(0)\right)<\psi_{2}\left(\tilde{x}_{2}\right)=0
$$

and

$$
v_{1}\left(\lambda_{2}, \frac{D}{D_{1}}\left(S_{\text {in }}-\lambda_{2}\right)\right)-D_{1}=\psi_{1}\left(\bar{x}_{1}(0)\right)<\psi_{1}\left(\tilde{x}_{1}\right)=0
$$

Hence, condition (45) is equivalent to condition (47).

\subsection{Modified Monod function}

We assume that the growth function $v_{i}$ is the modified Monod function

$$
v_{i}(S, x)=\frac{m_{i} S}{k_{i}+S+\beta_{i} x}, \quad i=1,2,
$$

where $m_{i}$ denotes the maximum growth rate, $k_{i}$ the half-saturation constant, and $\beta_{i}$ the inhibition factor for the growth of the species $i$ due to intra- and interspecific competition. This function contains both Monod's and Contois's functions as special cases (Roques et al. [38], Borja et al. [3]) and it was introduced in ecology by DeAngelis et al. [6] and by Beddington [2]. Such function is currently used in anaerobic digestion when the growth of a species is limited by one substrate while it is inhibited by another (cf. [49]). This growth function (49) satisfies the hypothesis (P1) and $(\mathrm{P} 2)$, as is easily checked. Straightforward calculations show that equations $f_{1}\left(x_{1}, x_{2}\right)=0$ and $f_{2}\left(x_{1}, x_{2}\right)=0$ define straight lines

$$
\begin{gathered}
x_{2}=\frac{\left[D D_{1}\left(k_{1}+S_{i n}\right)-D m_{1} S_{i n}\right]+\left[D_{1}\left(D \beta_{1}-D_{1}\right)+m_{1} D_{1}\right] x_{1}}{D_{1}\left(D_{2}-D \beta_{1} \alpha_{1}\right)-m_{1} D_{2}}, \\
x_{2}=\frac{\left[D D_{2}\left(k_{2}+S_{i n}\right)-D m_{2} S_{i n}\right]+\left[D_{2}\left(D \beta_{2} \alpha_{2}-D_{1}\right)+m_{2} D_{1}\right] x_{1}}{D_{2}\left(D_{2}-D \beta_{2}-m_{2}\right)} .
\end{gathered}
$$

Hence, these straight lines can have at most one intersection point. Therefore, the system can have at most one positive steady state, that is to say, Cases 1.b, 2.b, 3.b or 4.b depicted in Figures 2 and 3 are excluded. We have

$\bar{x}_{j}\left(\alpha_{i}\right)=\frac{D\left[D_{i}\left(k_{i}+S_{i n}\right)-m_{i} S_{i n}\right]}{D_{i}\left(D_{j}-D \beta_{i} \alpha_{i}\right)-m_{i} D_{j}}, \quad \tilde{x}_{i}=\frac{D}{D_{i}} \frac{\left[D_{i}\left(k_{i}+S_{i n}\right)-m_{i} S_{i n}\right]}{D_{i}-D \beta_{i}-m_{i}}, \quad \alpha_{i}^{c}=\frac{m_{i}\left(D S_{i n}-D_{j} \tilde{x}_{j}\right)-D_{i}\left(D\left(k_{i}+S_{i n}\right)-D_{j} \tilde{x}_{j}\right)}{D D_{i} \beta_{i} \tilde{x}_{j}}$.

Therefore, the results of Proposition 8 assert that for (37) where the $v_{i}$ are given by (49), the existence and stability of the positive steady states are as in Table 6. Numerical simulations using this function will be given in Section 7.

\begin{tabular}{c||c|c} 
& $0 \leqslant \alpha_{1}<\alpha_{1}^{c}$ & $\alpha_{1}^{c}<\alpha_{1}$ \\
\hline \hline $0 \leqslant \alpha_{2}<\alpha_{2}^{c}$ & Case 1.a & Case 4.a \\
$\alpha_{2}^{c}<\alpha_{2}$ & Case 3.a & Case 2.a
\end{tabular}

Table 6: Existence and stability of steady states of (37) according to $\alpha_{i}, i=1,2$, when the $v_{i}$ are given by (49). If, in addition, $D_{1}=D_{2}=D$ then Case 1.a should be replaced by Case 1.aS, that is, the positive steady state is stable. 


\section{Operating diagram}

In the following, we consider the system (4) when $D_{1}=D_{2}=D$. The aim of this section is to show the operating diagram that depicts regions in the $\left(S_{i n}, D\right)$-plane in which the system behavior changes by varying the two control parameters $S_{\text {in }}$ and $D$. All others parameters have biological meaning and they must be fixed since they depend on the organisms and substrate considered. Let $\Upsilon_{i}$ be the curve of equation $D=\mu_{i}\left(S_{i n}, 0,0\right), i=1,2$,

$$
\Upsilon_{i}=\left\{\left(S_{i n}, D\right): D=\mu_{i}\left(S_{i n}, 0,0\right)\right\} .
$$

Recall that $\tilde{x}_{i}$ and $\bar{x}_{i}$ are defined as the solutions of (11) and (22), respectively. These equations become now

$$
\mu_{i}\left(S_{\text {in }}-\tilde{x}_{i}, \tilde{x}_{i}, 0\right)=D, \quad \mu_{j}\left(S_{\text {in }}-\bar{x}_{i}, 0, \bar{x}_{i}\right)=D, \quad i=1,2, j=1,2, \quad i \neq j .
$$

Therefore, $\tilde{x}_{i}$ and $\bar{x}_{i}$ depend on the operating parameters $S_{i n}$ and $D$. We denote them by $\tilde{x}_{i}\left(S_{i n}, D\right)$ and $\bar{x}_{i}\left(S_{i n}, D\right)$. Define the sets

$$
\Upsilon_{i}^{c}=\left\{\left(S_{i n}, D\right): \tilde{x}_{i}\left(S_{i n}, D\right)=\bar{x}_{i}\left(S_{i n}, D\right)\right\},
$$

which are curves in the generic case. If the curves $\Upsilon_{i}, i=1,2$ intersect in a point $\left(S_{i n}^{*}, D^{*}\right)$ of the plane, then one has

$$
\mu_{1}\left(S_{i n}^{*}, 0,0\right)=\mu_{2}\left(S_{i n}^{*}, 0,0\right)=D^{*}
$$

From (50), we deduce that

$$
\tilde{x}_{i}\left(S_{i n}^{*}, D^{*}\right)=0 \quad \text { and } \quad \bar{x}_{i}\left(S_{i n}^{*}, D^{*}\right)=0, \quad i=1,2 .
$$

Therefore, the point $\left(S_{i n}^{*}, D^{*}\right)$ belongs to the sets $\Upsilon_{i}^{c}, i=1,2$. Hence, if $\Upsilon_{i}, i=1,2$ intersect in a point of $\left(S_{\text {in }}, D\right)$-plane, then the curves $\Upsilon_{i}^{c}$ intersect in the same point.

In the rest of this section, we restrict our attention to (37) where $D_{1}=D_{2}=D$ and the $v_{i}$ are given by (49). In this case, the set $\Upsilon_{i}^{c}$ is the curve of function $D=D_{i}^{c}\left(S_{\text {in }}\right)$ given by

$$
D_{i}^{c}\left(S_{i n}\right)=\frac{\eta_{0} S_{i n}+\eta_{1}}{\eta_{2} S_{\text {in }}+\eta_{3}}
$$

\begin{tabular}{|c|c|c|c|c|c|c|c|c|}
\hline Parameter & $m_{1}$ & $k_{1}$ & $m_{2}$ & $k_{2}$ & $\beta_{1}$ & $\beta_{2}$ & $\alpha_{1}$ & $\alpha_{2}$ \\
\hline Figure 7(a) & \multirow{4}{*}{4} & \multirow{4}{*}{1.5} & \multirow{4}{*}{2.2} & \multirow{4}{*}{2} & \multirow{4}{*}{1.2} & \multirow{4}{*}{0.2} & 0 & 0 \\
\hline Figure 7(b) & & & & & & & 0.7 & 0 \\
\hline Figures $7(\mathrm{c})$ and 10 & & & & & & & 0.7 & 1.73 \\
\hline Figure $7(d)$ & & & & & & & 0.7 & 2.5 \\
\hline Figure $8(a)$ & \multirow{4}{*}{2.5} & \multirow{4}{*}{1.5} & \multirow{4}{*}{1.2} & \multirow{4}{*}{0.2} & \multirow{4}{*}{1} & \multirow{4}{*}{2} & 0 & 0 \\
\hline Figure 8(b) & & & & & & & 3.8 & 0.27 \\
\hline Figures $8(\mathrm{c})$ and 11 & & & & & & & 4.58 & 0.27 \\
\hline Figure $8(d)$ & & & & & & & 4.58 & 0.35 \\
\hline Figure 9(a) & \multirow{4}{*}{2.5} & \multirow{4}{*}{1.5} & \multirow{4}{*}{1.2} & \multirow{4}{*}{0.2} & 0.3 & 0.35 & \multirow{4}{*}{0} & \multirow{4}{*}{0} \\
\hline Figure 9(b) & & & & & 0.1 & 0.06 & & \\
\hline Figure $9(\mathrm{c})$ & & & & & 0.01 & 0.01 & & \\
\hline Figure $9(d)$ & & & & & 0 & 0 & & \\
\hline
\end{tabular}

where

$$
\eta_{0}=\alpha_{j} \beta_{j} m_{i}-\beta_{i} m_{j}, \quad \eta_{1}=k_{j} m_{i}-k_{i} m_{j}, \quad \eta_{2}=\alpha_{j} \beta_{j}-\beta_{i}, \quad \eta_{3}=\alpha_{j} \beta_{j} k_{i}-\beta_{i} k_{j}-k_{i}+k_{j} .
$$

For the plot of the operating diagram, we must fix all biological parameters. The parameter values used for the simulations are provided in Table 7.

Table 7: Parameter values used for (37) where $D_{1}=D_{2}=D$ and the $v_{i}$ are given by (49). 
Figure 7 illustrates the case where the curves $\Upsilon_{1}$ and $\Upsilon_{2}$ do not intersect. In this case, the curves $\Upsilon_{i}$ and $\Upsilon_{i}^{c}, i=1,2$ separate the operating plane $\left(S_{i n}, D\right)$ in at most six regions, labeled as $I_{k}, k=0, \ldots, 5$. All regions appear in Figure 7(c). The regions $I_{4}$ and $I_{5}$ are empty in case (a) and the regions $I_{4}$ and $I_{3}$ are empty in case (b) and (d), respectively.

The transition from the region $\mathcal{I}_{0}$ to the region $\mathcal{I}_{1}$ by the curve $\Upsilon_{1}$ corresponds to a saddle-node bifurcation making the steady state $\mathcal{E}_{0}$ unstable (saddle point) with the appearance of an LES steady state $\mathcal{E}_{1}$. The transition from the region $\mathcal{I}_{1}$ to the region $\mathcal{I}_{2}$ by the curve $\Upsilon_{2}$ corresponds to the appearance of an unstable steady state $\mathcal{E}_{2}$ by a bifurcation with a saddle point $\mathcal{E}_{0}$. The transition from the region $\mathcal{I}_{2}$ to the region $\mathcal{I}_{3}$ by the curve $\Upsilon_{1}^{c}$ corresponds to a saddle-node bifurcation making the steady state $\mathcal{E}_{1}$ unstable with the appearance of an LES steady state $\mathcal{E}^{*}$. The transition from the region $\mathcal{I}_{2}$ to the region $\mathcal{I}_{4}$ by the curve $\Upsilon_{2}^{c}$ corresponds to a saddle-node bifurcation making the steady state $\mathcal{E}_{2}$ LES with the appearance of an unstable steady state $\mathcal{E}^{*}$. In this blue region $\mathcal{I}_{4}$, the system exhibits bistability of $\mathcal{E}_{1}$ and $\mathcal{E}_{2}$. The transition from the region $\mathcal{I}_{3}$ to the region $\mathcal{I}_{5}$ by the curve $\Upsilon_{2}^{c}$ corresponds to a saddle-node bifurcation of $\mathcal{E}_{2}$ and $\mathcal{E}^{*}$ making the steady state $\mathcal{E}_{2}$ LES with disappearance of the steady state $\mathcal{E}^{*}$. The transition from the region $\mathcal{I}_{4}$ to the region $\mathcal{I}_{5}$ by the curve $\Upsilon_{1}^{c}$ corresponds to a saddle-node bifurcation of $\mathcal{E}_{1}$ and $\mathcal{E}^{*}$ making the steady state $\mathcal{E}_{1}$ unstable with disappearance of the steady state $\mathcal{E}^{*}$.
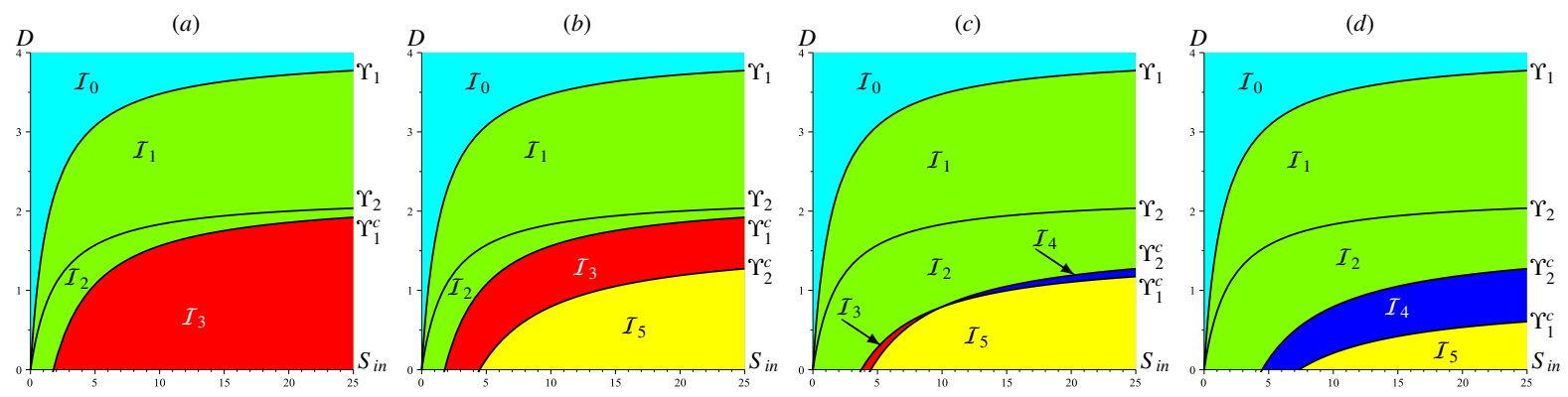

Figure 7: Operating diagrams of (37) where $D_{1}=D_{2}=D$ and the $v_{i}$ are given by (49) and the curves $\Upsilon_{i}$ do not intersect. The pictures show the occurrence of the bi-stability region $\mathcal{I}_{4}$ and the disappearance of the coexistence region $\mathcal{I}_{3}$ when $\alpha_{i}$ increase. In this figure and in Figures 8 and 9, cyan color represents the region of washout $\left(\mathcal{E}_{0}\right.$ is GAS), red color represents the region of coexistence ( $\mathcal{E}^{*}$ is GAS), blue color represents the region of bi-stability $\left(\mathcal{E}_{1}\right.$ and $\mathcal{E}_{2}$ are LES), green [resp. yellow] color represents a region of competitive exclusion $\left(\mathcal{E}_{1}\right.$ [resp. $\mathcal{E}_{2}$ ] is GAS).

Table 8 shows the existence and stability of steady states $\mathcal{E}_{0}, \mathcal{E}_{1}, \mathcal{E}_{2}$ and $\mathcal{E}^{*}$ in the regions $\mathcal{I}_{k}, k=0, \ldots, 5$, of the operating diagram, in Figure 7.

\begin{tabular}{lcccc}
\hline \hline Region & $\mathcal{E}_{0}$ & $\mathcal{E}_{1}$ & $\mathcal{E}_{2}$ & $\mathcal{E}^{*}$ \\
\hline$\left(S_{i n}, D\right) \in \mathcal{I}_{0}$ & $\mathrm{~S}$ & & & \\
$\left(S_{i n}, D\right) \in \mathcal{I}_{1}$ & $\mathrm{U}$ & $\mathrm{S}$ & & \\
$\left(S_{i n}, D\right) \in \mathcal{I}_{2}$ & $\mathrm{U}$ & $\mathrm{S}$ & $\mathrm{U}$ & \\
$\left(S_{i n}, D\right) \in \mathcal{I}_{3}$ & $\mathrm{U}$ & $\mathrm{U}$ & $\mathrm{U}$ & $\mathrm{S}$ \\
$\left(S_{i n}, D\right) \in \mathcal{I}_{4}$ & $\mathrm{U}$ & $\mathrm{S}$ & $\mathrm{S}$ & $\mathrm{U}$ \\
$\left(S_{i n}, D\right) \in \mathcal{I}_{5}$ & $\mathrm{U}$ & $\mathrm{U}$ & $\mathrm{S}$ & \\
\hline \hline
\end{tabular}

Table 8: Existence and local stability of steady states according to regions in the operating diagram of Figure 7, when the curves $\Upsilon_{i}$ do not intersect.

Figure 7(a) illustrates the operating diagram in the particular case studied by Lobry et al. [32, 34, 35] where $\alpha_{1}=\alpha_{2}=0$ (only the intraspecific competition terms are taken into account, see Section 5.2). In this case, if $D<\min \left(m_{1}, m_{2}\right)$ then, according to (44), coexistence occurs when $S_{\text {in }}$ is large enough. This result is illustrated by the red region of coexistence $\mathcal{I}_{3}$ in Figure 7(a). In order to maintain the coexistence of species, the ideal control parameter values of $D$ and $S_{\text {in }}$ should be chosen in $I_{3}$. Figure 7 shows how this region of coexistence changes and even disappears when interspecific competition is added in the model. For instance, if the first species interspecific coefficient $\alpha_{1}=0.7$ is fixed, then varying the second species interspecific coefficient $\alpha_{2}$ leads to a reduction of the coexistence region $\mathcal{I}_{3}$ until the occurrence of bi-stability region $\mathcal{I}_{4}$, followed by the disappearance of the coexistence region $\mathcal{I}_{3}$ (see Figure $7(\mathrm{a}-\mathrm{b}-\mathrm{c}-\mathrm{d})$ ). 
The case where curves $\Upsilon_{1}$ and $\Upsilon_{2}$ intersect is illustrated in Figure 8. We see in this figure that the curves $\Upsilon_{i}$ and $\Upsilon_{i}^{c}, i=1,2$ separate the operating plane $\left(S_{i n}, D\right)$ in seven regions, labeled as $\mathcal{I}_{k}, k=0, \ldots, 6$, see Figure $8(\mathrm{c})$. In Figure 8(a-b) [resp. (d)], the region $\mathcal{I}_{5}$ [resp. $\mathcal{I}_{4}$ ] is empty. Table 9 summarizes the existence and stability of steady states in these regions.

\begin{tabular}{lcccc}
\hline Region & $\mathcal{E}_{0}$ & $\mathcal{E}_{1}$ & $\mathcal{E}_{2}$ & $\mathcal{E}^{*}$ \\
\hline$\left(S_{i n}, D\right) \in \mathcal{I}_{0}$ & $\mathrm{~S}$ & & & \\
$\left(S_{i n}, D\right) \in \mathcal{I}_{1}$ & $\mathrm{U}$ & & $\mathrm{S}$ & \\
$\left(S_{i n}, D\right) \in \mathcal{I}_{2}$ & $\mathrm{U}$ & $\mathrm{S}$ & & \\
$\left(S_{i n}, D\right) \in \mathcal{I}_{3}$ & $\mathrm{U}$ & $\mathrm{S}$ & $\mathrm{U}$ & \\
$\left(S_{i n}, D\right) \in \mathcal{I}_{4}$ & $\mathrm{U}$ & $\mathrm{U}$ & $\mathrm{U}$ & $\mathrm{S}$ \\
$\left(S_{i n}, D\right) \in \mathcal{I}_{5}$ & $\mathrm{U}$ & $\mathrm{S}$ & $\mathrm{S}$ & $\mathrm{U}$ \\
$\left(S_{i n}, D\right) \in \mathcal{I}_{6}$ & $\mathrm{U}$ & $\mathrm{U}$ & $\mathrm{S}$ & \\
\hline \hline
\end{tabular}

Table 9: Existence and local stability of steady states according to regions in the operating diagram of Figure 8, when the curves $\Upsilon_{i}$ and $\Upsilon_{i}^{c}$ intersect.
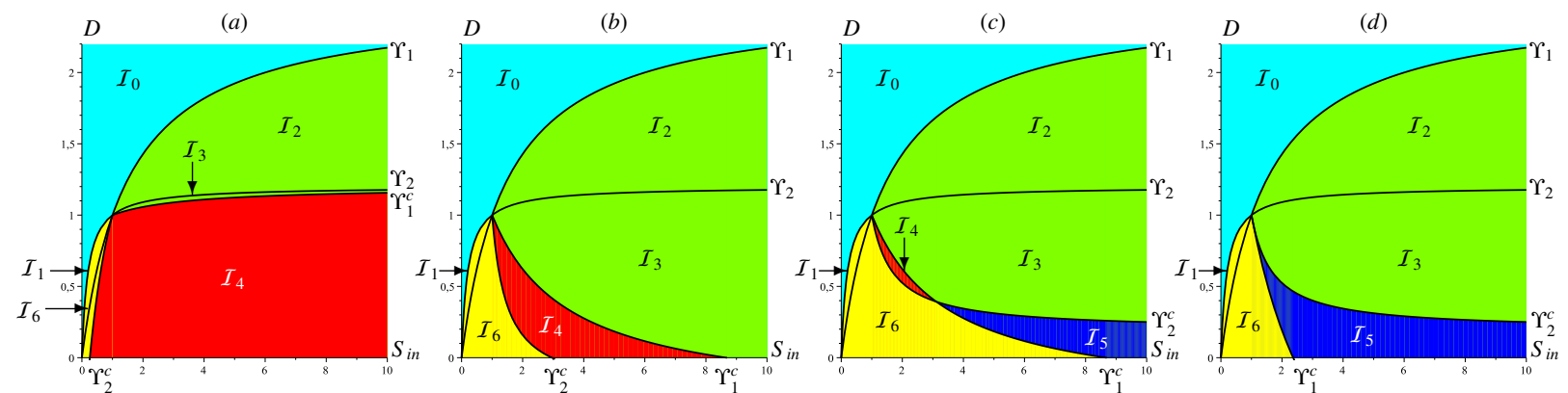

Figure 8: Operating diagrams of (37) where $D_{1}=D_{2}=D$ and the $v_{i}$ are given by (49) and the curves $\Upsilon_{i}$ intersect. The pictures show the occurrence of the bi-stability region $\mathcal{I}_{4}$ and the disappearance of the coexistence region $\mathcal{I}_{3}$ when $\alpha_{1}$ and $\alpha_{2}$ increase.
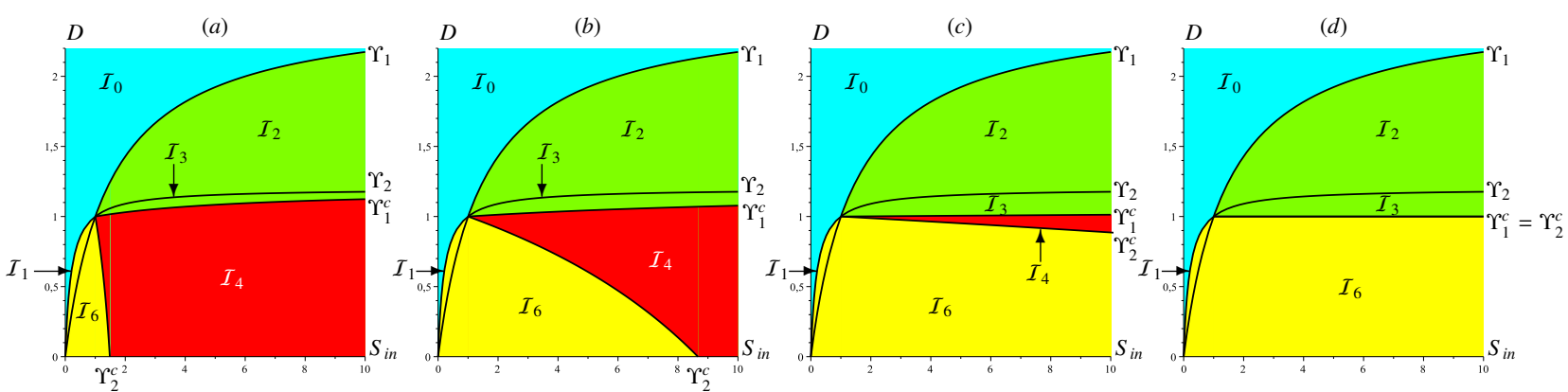

Figure 9: Operating diagrams of (37) where $D_{1}=D_{2}=D$ and the $v_{i}$ are given by (49) and the curves $\Upsilon_{i}$ intersect. The pictures show the deformation when $\beta_{1}$ and $\beta_{2}$ diminish, of the coexistence region $I_{4}$ in Figure 8(a), and its disappearance in the limiting case $\beta_{1}=\beta_{2}=0$ (panel d).

In the particular case $\alpha_{1}=\alpha_{2}=0$, from definition (51) of $D_{i}^{c}(\cdot)$, it follows that

$$
\lim _{S_{i n} \rightarrow+\infty} D_{i}^{c}\left(S_{i n}\right)=m_{j}
$$

Figure 8(a) illustrates the operating diagram in the particular case $\alpha_{1}=\alpha_{2}=0$. Recall that $D^{*}$ is defined by (50). If $D<D^{*}$ [resp. $D^{*}<D<\min \left(m_{1}, m_{2}\right)$ ], then increasing $S_{\text {in }}$ leads to the occurrence of an LES steady state $\mathcal{E}^{*}$ by a saddle-node bifurcation with the steady state $\mathcal{E}_{2}$ [resp. $\mathcal{E}_{1}$ ]. If $D>\min \left(m_{1}, m_{2}\right)$, the coexistence cannot occur for any value of the input nutrient concentration $S_{i n}$. Similarly to the previous case when the curves $\Upsilon_{i}$ do not intersect, increasing the interspecific competition parameters $\alpha_{i}$ introduces the bi-stability region $\mathcal{I}_{5}$ of boundary steady states $\mathcal{E}_{i}, i=1,2$ and disappears the coexistence region $\mathcal{I}_{4}$ (see Figure 8). 
Figure 9 shows how the coexistence region $\mathcal{I}_{4}$ is reduced when the intraspecific competition terms $\beta_{i}$ decrease to zero. In the limit case $\beta_{1}=\beta_{2}=0$, we obtain the operating diagram of the classical chemostat competition model which exhibits competitive exclusion, where only the species with the lowest break-even concentration survives (see Figure 9(d)). In this limit case $\beta_{1}=\beta_{2}=0$, it follows from (51) that the curves $\Upsilon_{i}^{c}$ of function $D=D_{i}^{c}\left(S_{i n}\right), i=1,2$ correspond simply to the straight line of equation

$$
D=\frac{k_{1} m_{2}-k_{2} m_{1}}{k_{1}-k_{2}}
$$

\section{Numerical simulations}

\subsection{Modified Monod function}

In this section, we consider again (37) where $D_{1}=D_{2}=D$ and the $v_{i}$ are given by (49). Recall that the existence and stability of the steady states are summarized in Table 6 , according to interspecific competition terms of two species $\alpha_{i}, i=1,2$. We plot the solutions of the corresponding reduced (32) in the variables $x_{1}$ and $x_{2}$. In order to illustrate the results of the previous section, we perform the simulations for the same biological parameter values that were considered for the plots of operating diagrams.

Consider the case where the curves $\Upsilon_{i}$ do not intersect (see Figure $7(\mathrm{c})$ ). We choose first $\left(S_{\text {in }}, D\right)=(6.5,0.46) \in$ $\mathcal{I}_{3}$. In this case, Figure 10 (a) illustrates the global convergence to the unique positive steady state $E^{*} \approx(2.393,3.191)$ which is GAS in the interior of $M$. This case corresponds to Case 1.aS in Table 6, or equivalently, $\alpha_{1}<\alpha_{1}^{c}$ and $\alpha_{2}<\alpha_{2}^{c}$. These conditions can be checked in Table 10. This Table summarizes the control parameter values $S_{\text {in }}$ and $D$ or equivalently the interspecific competition terms $\alpha_{i}$ and their critical values $\alpha_{i}^{c}$ in each case of Figure 10.

\begin{tabular}{l||c|c|c|c|c|c|c|c|c|c|c|c} 
& $\left(S_{\text {in }}, D\right)$ & Region & $\alpha_{1}$ & $\alpha_{1}^{c}$ & $\alpha_{2}$ & $\alpha_{2}^{c}$ & $\bar{x}_{1}(0)$ & $\bar{x}_{1}\left(\alpha_{2}\right)$ & $\tilde{x}_{1}$ & $\bar{x}_{2}(0)$ & $\bar{x}_{2}\left(\alpha_{1}\right)$ & $\tilde{x}_{2}$ \\
\hline \hline Figure 10(a) & $(6.5,0.46)$ & $I_{3}$ & 0.7 & 0.72 & 1.73 & 1.79 & 5.97 & 5.47 & 5.45 & 6.3 & 5.69 & 5.67 \\
Figure 10(b) & $(6.5,1)$ & $I_{2}$ & 0.7 & 1.12 & 1.73 & 0.77 & 4.83 & 3.75 & 4.29 & 6 & 4.69 & 4.14 \\
Figure 10(c) & $(20,0.9)$ & $I_{5}$ & 0.7 & 0.56 & 1.73 & 2.04 & 18.61 & 15.02 & 14.51 & 19.56 & 15.73 & 16.35 \\
Figure 10(d) & $(20,1.16)$ & $I_{4}$ & 0.7 & 0.68 & 1.73 & 1.64 & 17.77 & 12.82 & 13.01 & 19.39 & 14.43 & 14.53 \\
\hline Figure 11(a) & $(1.9,0.6)$ & $I_{4}$ & 4.58 & 4.80 & 0.27 & 0.28 & 1.7 & 1.1 & 1.08 & 1.43 & 0.58 & 0.57 \\
Figure 11(b) & $(1.9,0.9)$ & $I_{3}$ & 4.58 & 8.33 & 0.27 & 0.15 & 1.3 & 0.49 & 0.68 & 1.06 & 0.29 & 0.19 \\
Figure 11(c) & $(1.9,0.2)$ & $I_{6}$ & 4.58 & 3.82 & 0.27 & 0.36 & 1.86 & 1.68 & 1.63 & 1.77 & 1.27 & 1.33 \\
Figure 11(d) & $(5,0.25)$ & $I_{5}$ & 4.58 & 4.42 & 0.27 & 0.26 & 4.95 & 4.33 & 4.35 & 4.83 & 3.2 & 3.24
\end{tabular}

Table 10: Control parameter values and the interspecific competition terms used in Figures 10 and 11 .

(a)

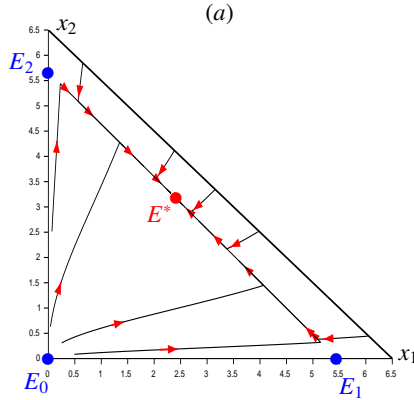

(b)

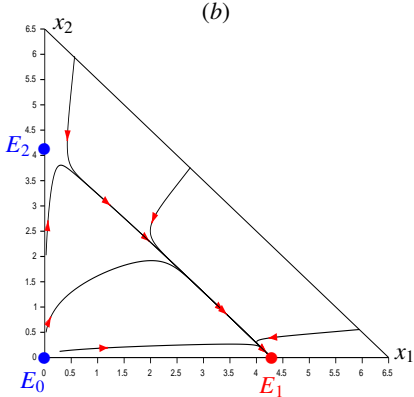

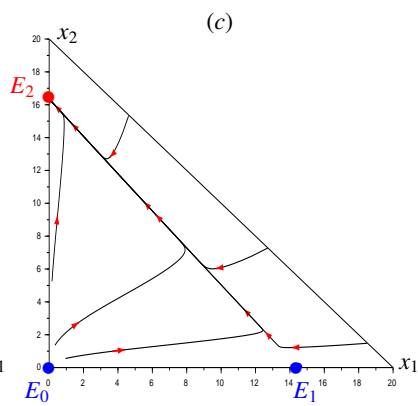

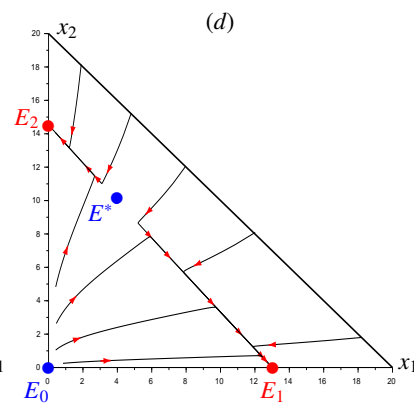

Figure 10: Phase portraits of (32) according to regions in the operating diagram of Figure 7(c): (a) Global convergence to $E^{*}$ for $\left(S_{\text {in }}, D\right) \in \mathcal{I}_{3}$. (b) Global convergence to $E_{1}$ for $\left(S_{i n}, D\right) \in \mathcal{I}_{2}$. (c) Global convergence to $E_{2}$ for $\left(S_{i n}, D\right) \in \mathcal{I}_{5}$. (d) Bi-stability of $E_{1}$ and $E_{2}$ for $\left(S_{i n}, D\right) \in \mathcal{I}_{4}$.

Figure 10(b) illustrates the global convergence to $E_{1}$ which is GAS in the interior of $M$ when $\left(S_{\text {in }}, D\right) \in \mathcal{I}_{2}$. Figure 10 (c) illustrates the global convergence to $E_{2}$ which is GAS in the interior of $M$ when $\left(S_{\text {in }}, D\right) \in \mathcal{I}_{5}$. Figure 10 (d) illustrates the bi-stability of $E_{1}$ and $E_{2}$ which are LES while $E^{*} \approx(3.952,10.05)$ and $E_{0}$ are unstable when $\left(S_{i n}, D\right) \in \mathcal{I}_{4}$. In each case, the coordinate $\tilde{x}_{i}$ of $E_{i}, i=1,2$ is given in Table 10 . 

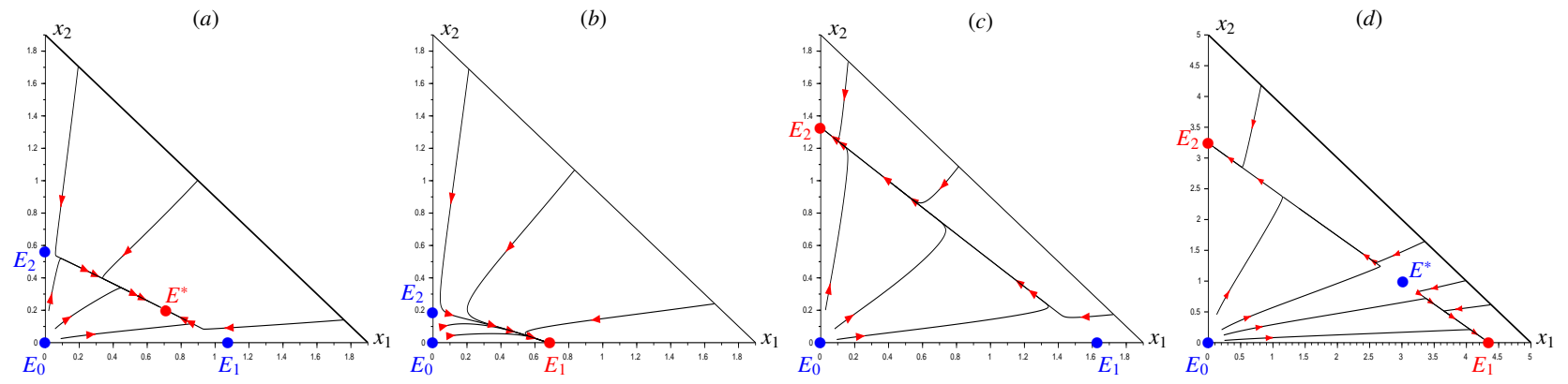

Figure 11: Phase portraits of (32) according to regions in the operating diagram of Figure 8(c): (a) Global convergence to $E^{*}$ for $\left(S_{\text {in }}, D\right) \in \mathcal{I}_{4}$. (b) Global convergence to $E_{1}$ for $\left(S_{i n}, D\right) \in \mathcal{I}_{3}$. (c) Global convergence to $E_{2}$ for $\left(S_{i n}, D\right) \in \mathcal{I}_{6}$. (d) Bi-stability of $E_{1}$ and $E_{2}$ for $\left(S_{i n}, D\right) \in \mathcal{I}_{5}$.

In order to show the result found in the operating diagram when the curves $\Upsilon_{i}$ and $\Upsilon_{i}^{c}$ intersect (see Figure 8(c)), we choose first $\left(S_{i n}, D\right) \in \mathcal{I}_{4}$. In this case, Figure 11(a) illustrates the global convergence to the unique positive steady state $E^{*} \approx(0.707,0.203)$ which is GAS in the interior of $M$. Figure 11(b) illustrates the global convergence to $E_{1}$ which is GAS in the interior of $M$ when $\left(S_{i n}, D\right) \in \mathcal{I}_{3}$. Figure 11(c) illustrates the global convergence to $E_{2}$ which is GAS in the interior of $M$ when $\left(S_{i n}, D\right) \in \mathcal{I}_{6}$. Figure 11(d) illustrates the bi-stability of $E_{1}$ and $E_{2}$ which are LES while $E^{*} \approx(3.022,0.978)$ and $E_{0}$ are unstable when $\left(S_{i n}, D\right) \in \mathcal{I}_{5}$.

\subsection{Generalized Monod function}

In this section, the existence of two positive steady states of (32) is illustrated with the following density-dependent growth rates, which are the product of a Monod function in $S$ by a decreasing function of $x$ :

$$
v_{i}(S, x)=\frac{m_{i} S}{k_{i}+S} \frac{1}{1+\beta_{i} x}, \quad i=1,2 .
$$

Such functions are currently used in biotechnology as the study of a syntrophic relationship in the anaerobic digestion process [41, 42]. We easily check that these functions (52) satisfy the properties (P1) and (P2). We succeeded in finding a set of parameters where there exist two positive steady states $E^{*}$ and $E^{* *}$ as provided in Table 11.

\begin{tabular}{c||c|c|c|c|c|c|c|c|c|c|c|c|c|c|c|c} 
Parameter & $m_{1}$ & $k_{1}$ & $m_{2}$ & $k_{2}$ & $\beta_{1}$ & $\beta_{2}$ & $D$ & $S_{\text {in }}$ & $\alpha_{1}$ & $\alpha_{1}^{c}$ & $\alpha_{2}$ & $\alpha_{2}^{c}$ & $\bar{x}_{1}\left(\alpha_{2}\right)$ & $\tilde{x}_{1}$ & $\bar{x}_{2}\left(\alpha_{1}\right)$ & $\tilde{x}_{2}$ \\
\hline \hline Figure 12 & 5.5 & 2 & 4.5 & 2.2 & 0.1 & 0.2 & 1.5 & 4.5 & 3.74 & 3.75 & 0.045 & 0.041 & 3.349 & 3.354 & 2.376 & 2.373
\end{tabular}

Table 11: Parameter values of (32) with the functions (52) used in Figure 12 where there exist two positive steady states.

Note that condition (45) is satisfied where $\bar{x}_{1}(0)=3.4$ and $\bar{x}_{2}(0)=3.75$ (see Table 11). Since $\tilde{x}_{2}<\tilde{x}_{1}$ and the condition $v_{1}\left(S_{\text {in }}-\tilde{x}_{2},+\infty\right)<D$ holds, there exist two critical values $\alpha_{i}^{c}, i=1,2$ provided in Table 11.
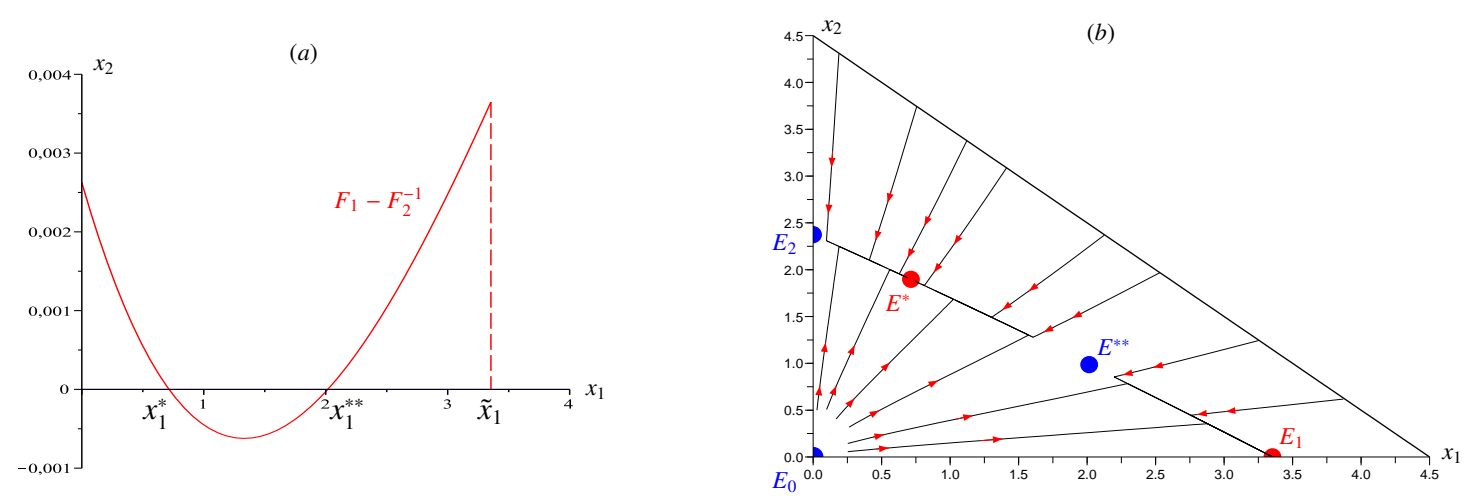

Figure 12: (a) Existence of two positive steady states $E^{*}$ and $E^{* *}$. (b) Bi-stability of $E^{*}$ and $E_{1}$. 
To illustrate the existence of two positive steady states, we simply represented the function $x_{1} \mapsto F_{1}\left(x_{1}\right)-F_{2}^{-1}\left(x_{1}\right)$ which vanishes at the abscissas of these two positive steady states (see Figure 12(a)). Figure 12(b) illustrates the Case 3S (see Table 4), that is, $1<\alpha_{1}<\alpha_{1}^{c}$ and $\alpha_{2}^{c}<\alpha_{2}<1$, where there exist two positive steady states

$$
E^{*} \approx(0.715,1.896) \text { and } \quad E^{* *} \approx(2.017,0.985) \text {. }
$$

In fact, for $1<\alpha_{1}<\alpha_{1}^{c}$ and by varying the parameter $\alpha_{2}$ from $\alpha_{2}<\alpha_{2}^{c}$ to $\alpha_{2}>\alpha_{2}^{c}$, an unstable positive steady state $E^{* *}$ appears with a saddle-node bifurcation when $\alpha_{2}=\alpha_{2}^{c}$ and then $E_{1}$ becomes LES. In this case, the system exhibits bi-stability of $E^{*}$ and $E_{1}$ which are LES while $E_{0}, E_{2}$ and $E^{* *}$ are unstable.

\section{Conclusion}

In this work, we have analyzed the effect of the intra- and interspecific competition on the coexistence of microbial species in a chemostat. In this context, we have studied the general density-dependent model (4) of two species where the dilution rates are distinct and the growth rates depend on the substrate $S$ and the two species concentrations $x_{i}$, $i=1,2$. The functions are assumed to be increasing in the substrate $S$ and decreasing in each species concentration $x_{i}, i=1,2$ due to the effect of intra- and interspecific competition.

We give the condition of existence and local stability of all corresponding steady states of (4). We define four numbers $\bar{x}_{i}$ and $\tilde{x}_{i}, i=1,2$ and, according to their relative positions, we distinguish four cases, (23) and (24), which are qualitatively similar to the cases encountered in the classical Lotka-Volterra competition model. When the intraspecific competition is dominant with respect to interspecific competition there exists at most one positive steady state, that is LES, and the model exhibits the coexistence of the species. Inversely, when the intraspecific competition is dominated by interspecific competition, there exists at most one positive steady state, that is unstable and the model exhibits bistability.

In the particular case $D_{1}=D_{2}=D$, three order model (4) can be reduced to second order model (32), thanks to the mass conservation principle. Moreover, due to Thieme's results [47], the global asymptotic stability of each steady state of (4) is derived from that of reduced model (32).

The density-dependent growth rates (5) are considered and the dependence of the existence and stability of steady states of (37), with respect to the interspecific competition parameters $\alpha_{i}$, is investigated. In the particular case of (37) with only the intraspecific competition, that is, $\alpha_{1}=0$ and $\alpha_{2}=0$, our results are compared with those obtained by Lobry et al. [33-35] by using the concept of the steady-state characteristic. More precisely, we show that the existence condition (44) of the positive steady state $\mathcal{E}^{*}$, obtained by Lobry et al. $[34,35]$ in the particular case $D_{1}=D_{2}=D$, is equivalent to Case 1 or to our condition (47).

The operating diagrams show how regions of coexistence and bi-stability of steady states vary with intra- and interspecific competition. They demonstrate that competition has a significant impact on the maintenance of species coexistence and the protection of the least relevant species among microbial ecosystems. More precisely, increasing the values of interspecific competition terms reduces the region of coexistence and increases the regions of competitive exclusion with the occurrence of a bi-stability region. Indeed, we believe that the originality of our work is to bring out the common effects of the intra- and interspecific competition to explain the emergence of coexistence.

Our study reveals one main characteristic of intra- and interspecific density-dependent model: For small enough interspecific competition terms, there is a stable persistence of two species which can coexist for any positive initial condition. However, if these terms are large enough, the system exhibits bi-stability with the competitive exclusion of one species according to the initial condition. The simulations illustrate the mathematical results demonstrated in the case where the density-dependent growth rates are of modified Monod-type (49) or of generalized Monod-type (52).

\section{Appendix A. Proofs}

Proof of Prop. 4. From (21) we deduce that

$$
F_{1}^{\prime}=-\frac{\frac{D_{1}}{D} \frac{\partial \mu_{1}}{\partial S}-\frac{\partial \mu_{1}}{\partial x_{1}}}{\frac{D_{2}}{D} \frac{\partial \mu_{1}}{\partial S}-\frac{\partial \mu_{1}}{\partial x_{2}}}=-\frac{D_{1}}{D_{2}} \frac{\frac{D_{1}}{D} \frac{\partial \mu_{1}}{\partial S}-\frac{\partial \mu_{1}}{\partial x_{1}}}{\frac{D_{1}}{D} \frac{\partial \mu_{1}}{\partial S}-\frac{D_{1}}{D_{2}} \frac{\partial \mu_{1}}{\partial x_{2}}} .
$$


Using (27) we deduce that $F_{1}^{\prime}<-\frac{D_{1}}{D_{2}}$. From (21) we deduce that

$$
F_{2}^{\prime}=-\frac{\frac{D_{2}}{D} \frac{\partial \mu_{2}}{\partial S}-\frac{\partial \mu_{2}}{\partial x_{2}}}{\frac{D_{1}}{D} \frac{\partial \mu_{2}}{\partial S}-\frac{\partial \mu_{2}}{\partial x_{1}}}=-\frac{D_{2}}{D_{1}} \frac{\frac{D_{2}}{D} \frac{\partial \mu_{2}}{\partial S}-\frac{\partial \mu_{2}}{\partial x_{2}}}{\frac{D_{2}}{D} \frac{\partial \mu_{2}}{\partial S}-\frac{D_{2}}{D_{1}} \frac{\partial \mu_{2}}{\partial x_{1}}} .
$$

Using (27) we deduce that $F_{2}^{\prime}<-\frac{D_{2}}{D_{1}}$. Consequently, if (27) holds then the curves $\gamma_{1}$ and $\gamma_{2}$ can intersect at most once, since at each intersection point we have

$$
F_{1}^{\prime} F_{2}^{\prime}>\left(-\frac{D_{1}}{D_{2}}\right)\left(-\frac{D_{1}}{D_{2}}\right)=1
$$

Thus, if Case 1 holds, there is a unique intersection and if Case 3 or Case 4 hold, then there is no intersection. If $\bar{x}_{1}<\tilde{x}_{1}$, then necessarily $\bar{x}_{2}>\tilde{x}_{2}$, so that Case 2 cannot hold.

Similarly, using (A.1), (A.2) and (28) we prove that $F_{1}^{\prime}>-\frac{D_{1}}{D_{2}}$ and $F_{2}^{\prime}>-\frac{D_{2}}{D_{1}}$. Therefore, if (28) holds then the curves $\gamma_{1}$ and $\gamma_{2}$ can intersect at most once, since at each intersection point we have $F_{1}^{\prime} F_{2}^{\prime}<1$. Thus, if Case 2 holds, there is a unique intersection and if Case 3 or Case 4 hold, then there is no intersection. If $\bar{x}_{1}>\tilde{x}_{1}$, then necessarily $\bar{x}_{2}<\tilde{x}_{2}$, so that Case 1 cannot hold.

For the stability analysis, it is useful to use the change of variables $z=S+x_{1}+x_{2}$ introduced in Proposition 1 . Using the variables $\left(z, x_{1}, x_{2}\right)$, (4) can be written

$$
\left\{\begin{aligned}
\dot{z} & =D\left(S_{\text {in }}-z\right)-a_{1} x_{1}-a_{2} x_{2} \\
\dot{x}_{1} & =\left[\mu_{1}\left(z-x_{1}-x_{2}, x_{1}, x_{2}\right)-D_{1}\right] x_{1} \\
\dot{x}_{2} & =\left[\mu_{2}\left(z-x_{1}-x_{2}, x_{2}, x_{1}\right)-D_{2}\right] x_{2}
\end{aligned}\right.
$$

where $a_{1}=D_{1}-D \geqslant 0$ and $a_{2}=D_{2}-D \geqslant 0$. The Jacobian matrix of (A.3) at $\left(z, x_{1}, x_{2}\right)$ is

$$
J=\left[\begin{array}{ccc}
-D & -a_{1} & -a_{2} \\
m_{10} & -m_{11}+\mu_{1}-D_{1} & -m_{12} \\
m_{20} & -m_{21} & -m_{22}+\mu_{2}-D_{2}
\end{array}\right] .
$$

where

$$
\begin{aligned}
& m_{10}=\frac{\partial \mu_{1}}{\partial S} x_{1}, \quad m_{11}=\left(\frac{\partial \mu_{1}}{\partial S}-\frac{\partial \mu_{1}}{\partial x_{1}}\right) x_{1}, \quad m_{12}=\left(\frac{\partial \mu_{1}}{\partial S}-\frac{\partial \mu_{1}}{\partial x_{2}}\right) x_{1}, \\
& m_{20}=\frac{\partial \mu_{2}}{\partial S} x_{2}, \quad m_{21}=\left(\frac{\partial \mu_{2}}{\partial S}-\frac{\partial \mu_{2}}{\partial x_{1}}\right) x_{2}, \quad m_{22}=\left(\frac{\partial \mu_{2}}{\partial S}-\frac{\partial \mu_{2}}{\partial x_{2}}\right) x_{2},
\end{aligned}
$$

are evaluated at $\left(z, x_{1}, x_{2}\right)$. They are nonnegative.

Proof of Prop. 5. At $\mathcal{E}_{0}$, the Jacobian matrix (A.4) is given by

$$
J_{0}=\left[\begin{array}{ccc}
-D & -a_{1} & -a_{2} \\
0 & \mu_{1}\left(S_{i n}, 0,0\right)-D_{1} & 0 \\
0 & 0 & \mu_{2}\left(S_{i n}, 0,0\right)-D_{2}
\end{array}\right]
$$

The eigenvalues are negative if and only if $\mu_{i}\left(S_{i n}, 0,0\right)<D_{i}$, for $i=1,2$. Using Proposition 2, we deduce that these conditions hold if and only if the boundary steady states $\mathcal{E}_{i}, i=1,2$ do not exist.

At $\mathcal{E}_{1}$, the Jacobian matrix (A.4) is given by

$$
J_{1}=\left[\begin{array}{ccc}
-D & -a_{1} & -a_{2} \\
m_{10} & -m_{11} & -m_{12} \\
0 & 0 & f_{2}\left(\tilde{x}_{1}, 0\right)
\end{array}\right] .
$$

The eigenvalues are

$$
f_{2}\left(\tilde{x}_{1}, 0\right)=\mu_{2}\left(S_{\text {in }}-\frac{D_{1}}{D} \tilde{x}_{1}, 0, \tilde{x}_{1}\right)-D_{2}
$$


which is negative if and only if $\tilde{x}_{1}>\bar{x}_{1}$, together with the eigenvalues of the upper left square matrix

$$
A_{1}=\left[\begin{array}{cc}
-D & -a_{1} \\
m_{10} & -m_{11}
\end{array}\right]
$$

Since

$$
\operatorname{tr} A_{1}=-D-m_{11}<0, \quad \operatorname{det} A_{1}=D m_{11}+a_{1} m_{10}>0,
$$

the real part of the eigenvalues of $A_{1}$ are negative. Therefore, $\mathcal{E}_{1}$ is LES if and only if $\tilde{x}_{1}>\bar{x}_{1}$. Similarly, at $\mathcal{E}_{2}$, the Jacobian matrix (A.4) is given by

$$
J_{2}=\left[\begin{array}{ccc}
-D & -a_{1} & -a_{2} \\
0 & f_{1}\left(0, \tilde{x}_{2}\right) & 0 \\
m_{20} & -m_{21} & -m_{22}
\end{array}\right] .
$$

The eigenvalues are

$$
f_{1}\left(0, \tilde{x}_{2}\right)=\mu_{1}\left(S_{\text {in }}-\frac{D_{2}}{D} \tilde{x}_{2}, 0, \tilde{x}_{2}\right)-D_{1},
$$

which is negative if and only if $\tilde{x}_{2}>\bar{x}_{2}$, together with the eigenvalues of the square sub-matrix

$$
A_{2}=\left[\begin{array}{cc}
-D & -a_{2} \\
m_{20} & -m_{22}
\end{array}\right]
$$

Since

$$
\operatorname{tr} A_{2}=-D-m_{22}<0, \quad \operatorname{det} A_{2}=D m_{22}+a_{2} m_{20}>0,
$$

the real part of the eigenvalues of $A_{2}$ are negative. Therefore, $\mathcal{E}_{2}$ is LES if and only if $\tilde{x}_{2}>\bar{x}_{2}$.

Proof of Prop. 6. At $\mathcal{E}^{*}$, the Jacobian matrix (A.4) is given by

$$
J^{*}=\left[\begin{array}{ccc}
-D & -a_{1} & -a_{2} \\
m_{10} & -m_{11} & -m_{12} \\
m_{20} & -m_{21} & -m_{22}
\end{array}\right] .
$$

The characteristic polynomial of $J^{*}$ is given by $P(\lambda)=-\lambda^{3}+c_{1} \lambda^{2}+c_{2} \lambda+c_{3}$, where

$$
c_{1}=-D-m_{11}-m_{22}, \quad c_{2}=m_{12} m_{21}-m_{11} m_{22}-D\left(m_{11}+m_{22}\right)-a_{1} m_{10}-a_{2} m_{20}
$$

and

$$
c_{3}=D\left(m_{12} m_{21}-m_{11} m_{22}\right)+a_{1}\left(m_{12} m_{20}-m_{10} m_{22}\right)+a_{2}\left(m_{10} m_{21}-m_{11} m_{20}\right) .
$$

Since $c_{1}<0$, according to the Routh-Hurwitz criterion, $\mathcal{E}^{*}$ is LES if and only if

$$
c_{2}<0, \quad c_{3}<0, \quad c_{1} c_{2}+c_{3}>0
$$

Using the expressions of $m_{i k}, i=1,2, k=0,1,2$ given by (A.5,A.6), the derivatives of the functions $F_{i}(\cdot)$ can be written as

$$
F_{1}^{\prime}=-\frac{a_{1} m_{10}+D m_{11}}{a_{2} m_{10}+D m_{12}}, \quad F_{2}^{\prime}=-\frac{a_{2} m_{20}+D m_{22}}{a_{1} m_{20}+D m_{21}} .
$$

As a consequence one has,

$$
1-F_{1}^{\prime} F_{2}^{\prime}=\frac{D^{2}\left(m_{12} m_{21}-m_{11} m_{22}\right)+D a_{1}\left(m_{12} m_{20}-m_{10} m_{22}\right)+D a_{2}\left(m_{10} m_{21}-m_{11} m_{20}\right)}{\left(a_{2} m_{10}+D m_{12}\right)\left(a_{1} m_{20}+D m_{21}\right)} .
$$

Thus,

$$
c_{3}=\left[1-F_{1}^{\prime}\left(x_{1}^{*}\right) F_{2}^{\prime}\left(x_{2}^{*}\right)\right]\left(a_{2} m_{10}+D m_{12}\right)\left(a_{1} m_{20}+D m_{21}\right) / D
$$


Therefore, $c_{3}<0$ if and only if $F_{1}^{\prime}\left(x_{1}^{*}\right) F_{2}^{\prime}\left(x_{2}^{*}\right)>1$. This proves that $\mathcal{E}^{*}$ is unstable if $F_{1}^{\prime}\left(x_{1}^{*}\right) F_{2}^{\prime}\left(x_{2}^{*}\right)<1$, which is item 1 of the proposition.

For the proof of item 2, we consider first the case where (26) holds. A straightforward calculation shows that

$$
\begin{aligned}
c_{1} c_{2}+c_{3}= & \left(m_{11}+m_{22}\right)\left(m_{11} m_{22}-m_{12} m_{21}\right)+D\left(D\left(m_{11}+m_{22}\right)+\left(m_{11}+m_{22}\right)^{2}+a_{1} m_{10}+a_{2} m_{20}\right) \\
& +a_{1}\left(m_{12} m_{20}+m_{10} m_{11}\right)+a_{2}\left(m_{10} m_{21}+m_{20} m_{22}\right) .
\end{aligned}
$$

We have

$$
m_{11} m_{22}-m_{12} m_{21}=\left[\frac{\partial \mu_{1}}{\partial S}\left(\frac{\partial \mu_{2}}{\partial x_{1}}-\frac{\partial \mu_{2}}{\partial x_{2}}\right)+\frac{\partial \mu_{2}}{\partial S}\left(\frac{\partial \mu_{1}}{\partial x_{2}}-\frac{\partial \mu_{1}}{\partial x_{1}}\right)+\frac{\partial \mu_{1}}{\partial x_{1}} \frac{\partial \mu_{2}}{\partial x_{2}}-\frac{\partial \mu_{1}}{\partial x_{2}} \frac{\partial \mu_{2}}{\partial x_{1}}\right] x_{1}^{*} x_{2}^{*}
$$

Using (26) and (H2) we deduce that

$$
\frac{\partial \mu_{1}}{\partial x_{1}} \frac{\partial \mu_{2}}{\partial x_{2}}>\frac{\partial \mu_{1}}{\partial x_{2}} \frac{\partial \mu_{2}}{\partial x_{1}}
$$

Hence, $m_{11} m_{22}-m_{12} m_{21}>0$, and we conclude that if (26) holds, then $c_{2}<0$ and $c_{1} c_{2}+c_{3}>0$, that is, conditions (A.7) are satisfied if and only if $F_{1}^{\prime}\left(x_{1}^{*}\right) F_{2}^{\prime}\left(x_{2}^{*}\right)>1$.

Let us prove now item 2 in the case where $D_{1}=D_{2}=D$. Since $a_{1}=a_{2}=0$, the Jacobian matrix (A.4) is given by

$$
J^{*}=\left[\begin{array}{ccc}
-D & 0 & 0 \\
m_{10} & -m_{11} & -m_{12} \\
m_{20} & -m_{21} & -m_{22}
\end{array}\right] .
$$

The eigenvalues are $-D$ together with the eigenvalues of the square sub-matrix

$$
A^{*}=\left[\begin{array}{ll}
-m_{11} & -m_{12} \\
-m_{21} & -m_{22}
\end{array}\right]
$$

We have

$$
\operatorname{tr} A^{*}=-m_{11}-m_{22}<0, \quad \operatorname{det} A^{*}=m_{11} m_{22}-m_{12} m_{21} .
$$

Replace $a_{1}$ and $a_{2}$ by zero in (A.8), yields

$$
F_{1}^{\prime}\left(x_{1}^{*}\right) F_{2}^{\prime}\left(x_{2}^{*}\right)-1=\frac{m_{11} m_{22}-m_{12} m_{21}}{m_{12} m_{21}} .
$$

Thus,

$$
\operatorname{det} A^{*}=\left[F_{1}^{\prime}\left(x_{1}^{*}\right) F_{2}^{\prime}\left(x_{2}^{*}\right)-1\right] m_{12} m_{21} .
$$

Therefore, $\mathcal{E}^{*}$ is LES if and only if $F_{1}^{\prime}\left(x_{1}^{*}\right) F_{2}^{\prime}\left(x_{2}^{*}\right)>1$. This completes the proof of item 2 of the proposition.

\section{Acknowledgments}

We thank the financial support of PHC UTIQUE project No. 13G1120 and of TREASURE euro-Mediterranean research network (https://project.inria.fr/treasure/).

\section{References}

[1] N. Abdellatif, R. Fekih-Salem and T. Sari, Competition for a single resource and coexistence of several species in the chemostat, Math. Biosci. Eng., 13 (2016), 631-652.

[2] J. R. Beddington, Mutual interference between parasites or predators and its effect on searching efficiency, J. Anim. Ecol., 44 (1975), 331-340.

[3] R. Borja, C. J. Banks, A. Martin and B. Khalfaoui, Anaerobic digestion of palm oil mill effluent and condensation water waste: an overall kinetic model for methane production and substrate utilization, Bioprocess. Eng., 13 (1995), 87-95.

[4] J. P. Braselton and P. Waltman, A competition model with dynamically allocated inhibitor production. Math. Biosci., 173 (2001), 55-84. 
[5] R. S. Cantrell, C. Cosner and S. Ruan, Intraspecific interference and consumer-resource dynamics, Discrete and Continuous Dynamical Systems-Series B, 4 (2004), 527-546.

[6] D. L. DeAngelis, R. A. Goldstein and R. V. O'Neill, A model for trophic interaction, Ecology, 56 (1975), 881-892.

[7] R. Fekih-Salem, Modèles mathématiques pour la compétition et la coexistence des espèces microbiennes dans un chémostat, (Ph.D. thesis), University of Montpellier 2 and University of Tunis el Manar, 2013. https ://tel.archives-ouvertes.fr/tel-01018600.

[8] R. Fekih-Salem, J. Harmand, C. Lobry, A. Rapaport and T. Sari, Extensions of the chemostat model with flocculation, J. Math. Anal. Appl., 397 (2013), 292-306.

[9] R. Fekih-Salem, A. Rapaport and T. Sari, Emergence of coexistence and limit cycles in the chemostat model with flocculation for a general class of functional responses, Appl. Math. Modell., 40 (2016), 7656-7677.

[10] R. Fekih-Salem and T. Sari, Sur la stabilité globale de l'équilibre de coexistence d'un modèle densité-dépendant de compétition pour une ressource, Proceedings of the 12th African Conference on Research in Computer Science and Applied Mathematics, INRIA (2014), 19-30.

[11] R. Fekih-Salem, T. Sari and N. Abdellatif, Sur un modèle de compétition et de coexistence dans le chémostat, ARIMA J., 14 (2011), 15-30.

[12] H. Fgaier, M. Kalmokoff, T. Ells and H. J. Eberl, An allelopathy based model for the Listeria overgrowth phenomenon, Math. Biosci., 247 (2014), 13-26.

[13] J. P. Grover and F. B. Wang, Competition for one nutrient with internal storage and toxin mortality, Math. Biosci., 244 (2013), 82-90.

[14] B. Haegeman, C. Lobry and J. Harmand, Modeling bacteria flocculation as density-dependent growth, AIChE J., 53 (2007), $535-539$.

[15] B. Haegeman and A. Rapaport, How flocculation can explain coexistence in the chemostat, J. Biol. Dyn., 2 (2008), 1-13.

[16] S.R. Hansen and S.P. Hubbell, Single-nutrient microbial competition: qualitative agreement between experimental and theoretically forecast outcomes, Science, 207 (1980), 1491-1493.

[17] G. Hardin, The competitive exclusion principle, Science, 131 (1960), 1292-1297.

[18] J. Harmand, A. Rapaport, D. Dochain and C. Lobry, Microbial ecology and bioprocess control: Opportunities and challenges, Journal of Process Control, 18 (2008), 865-875.

[19] J. Heßeler, J.K. Schmidt, U. Reichl and D. Flockerzi, Coexistence in the chemostat as a result of metabolic by-products, J. Math. Biol., 53 (2006), 556-584.

[20] M.W. Hirsch and S. Smale, Differential Equations, Dynamical Systems, and Linear Algebra. Academic Press, Inc, 1974.

[21] S. B. Hsu, Limiting behavior for competing species, SIAM J. Appl. Math, 34 (1978), 760-763.

[22] S. B. Hsu, S. P. Hubbell and P. Waltman, A mathematical theory for single-nutrient competition in continuous cultures of micro-organisms, SIAM J. Appl. Math., 32 (1977), 366-383.

[23] S. B. Hsu, Y. S. Li and P. Waltman, Competition in the presence of a lethal external inhibitor, Math. Biosci., 167 (2000), $177-199$.

[24] S. B. Hsu and P. Waltman, Analysis of a model of two competitors in a chemostat with an external inhibitor, SIAM J. Appl. Math, 52 (1992), $528-540$.

[25] S. B. Hsu and P. Waltman, A survey of mathematical models of competition with an inhibitor, Math. Biosci., 187 (2004), 53-91.

[26] G. E. Hutchinson, The paradox of the plankton, Am. Nat., 95 (1961), 137-145.

[27] L. Imhof and S. Walcher, Exclusion and persistence in deterministic and stochastic chemostat models, J. Differential Equations, 217 (2005), 26-53.

[28] P. De Leenheer, D. Angeli and E. D. Sontag, Crowding effects promote coexistence in the chemostat, J. Math. Anal. Appl., 319 (2006), 48-60.

[29] P. De Leenheer, B. Li and H. L. Smith, Competition in the chemostat: Some remarks, Canadian Applied Mathematics Quarterly, 11 (2003), 229-248.

[30] R. E. Lenski and S. E. Hattingh, Coexistence of two competitors on one resource and one inhibitor: a chemostat model based on bacteria and antibiotics, J. Theor. Biol., 122 (1986), 83-93.

[31] B. Li, Global asymptotic behavior of the chemostat: general response functions and different removal rates, SIAM J. Appl. Math., 59 (1998), 411-422.

[32] C. Lobry and J. Harmand, A new hypothesis to explain the coexistence of $n$ species in the presence of a single resource, C. R. Biol., 329 (2006), 40-46.

[33] C. Lobry and F. Mazenc, Effect on persistence of intra-specific competition in competition models, Electron. J. Diff. Eqns., 125 (2007), 1-10.

[34] C. Lobry, F. Mazenc and A. Rapaport, Persistence in ecological models of competition for a single resource, C. R. Acad. Sci. Paris, Ser. I, 340 (2005), 199-204.

[35] C. Lobry, A. Rapaport and F. Mazenc, Sur un modèle densité-dépendant de compétition pour une ressource, C. R. Biol., 329 (2006), 63-70.

[36] J. Monod, La technique de culture continue. Théorie et applications, Ann. Inst. Pasteur, 79 (1950), 390-410.

[37] A. Rescigno and I. W. Richardson, The struggle for life: I. Two species, Bull. Math. Biophys., 29 (1967), $377-388$.

[38] H. Roques, S. Yue, S. Saipanich and B. Capdeville, Faut-il abandonner le formalisme de Monod pour la modélisation des processus de dépollution par voie biologique?, Water Res., 16 (1982), 839-847.

[39] S. Ruan, A. Ardito, P. Ricciardi and D. L. DeAngelis, Coexistence in competition models with density-dependent mortality, C. R. Biol., 330 (2007), 845-854.

[40] Y. Saito and T. Miki, Species coexistence under resource competition with intraspecific and interspecific direct competition in a chemostat, Theor. Popul. Biol., 78 (2010), 173-182. 
[41] T. Sari, M. El Hajji and J. Harmand, The mathematical analysis of a syntrophic relationship between two microbial species in a chemostat, Math. Biosci. Eng., 9 (2012), 627-645.

[42] T. Sari and J. Harmand, A model of a syntrophic relationship between two microbial species in a chemostat including maintenance, Math. Biosci., 275 (2016), 1-9.

[43] T. Sari and F. Mazenc, Global dynamics of the chemostat with different removal rates and variable yields, Math. Biosci. Eng., 8 (2011), $827-840$.

[44] M. Scheffer, S. Rinaldi, J. Huisman and F.J. Weissing, Why plankton communities have no equilibrium: solutions to the paradox, Hydrobiologia, 491 (2003), 9-18.

[45] J. K. Schmidt, B. König and U. Reichl, Characterization of a three bacteria mixed culture in a chemostat: evaluation and application of a quantitative Terminal-Restriction Fragment Length Polymorphism (T-RFLP) analysis for absolute and species specific cell enumeration, Biotechnol. Bioeng., 96 (2007), 738-756.

[46] H. L. Smith and P. Waltman, The Theory of the Chemostat: Dynamics of Microbial Competition, Cambridge University Press, 1995.

[47] H. R. Thieme, Convergence results and a Poincaré-Bendixson trichotomy for asymptotically autonomous differential equations, J. Math. Biol., 30 (1992), 755-763.

[48] M.J. Wade, R.W. Pattinson, N. G. Parker and J. Dolfing, Emergent behaviour in a chlorophenol-mineralising three-tiered microbial 'food web', J. Theor. Biol., 389 (2016), 171-186.

[49] M. Weedermann, G. Seo and G.S.K. Wolkowicz, Mathematical model of anaerobic digestion in a chemostat: effects of syntrophy and inhibition, J. Biol. Dyn., 7 (2013), 59-85.

[50] G. S. K. Wolkowicz and Z. Lu, Global dynamics of a mathematical model of competition in the chemostat: general response functions and differential death rates, SIAM J. Appl. Math., 52 (1992), 222-233.

[51] G. S. K. Wolkowicz and Z. Lu, Direct interference on competition in a chemostat, J. Biomath, 13 (1998), 282-291. 